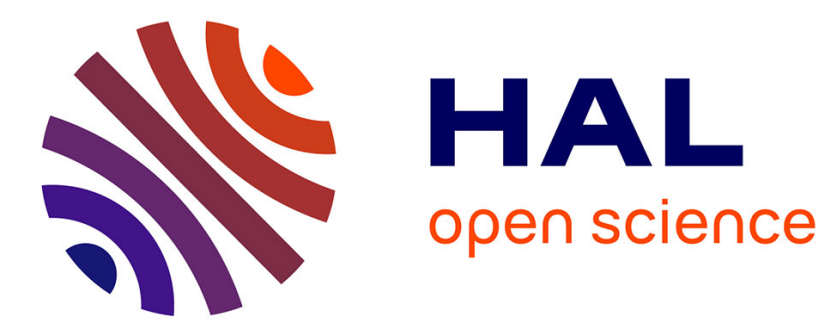

\title{
Parametric instability in a rotating cylinder of gas subject to sinusoidal axial compression. Part 2. Weakly nonlinear theory
}

Jean-Philippe Racz, Julian F. Scott

\section{- To cite this version:}

Jean-Philippe Racz, Julian F. Scott. Parametric instability in a rotating cylinder of gas subject to sinusoidal axial compression. Part 2. Weakly nonlinear theory. Journal of Fluid Mechanics, 2008, 595, pp.291-321. 10.1017/S0022112007009226 . hal-00445037

\section{HAL Id: hal-00445037 \\ https://hal.science/hal-00445037}

Submitted on 13 Jan 2010

HAL is a multi-disciplinary open access archive for the deposit and dissemination of scientific research documents, whether they are published or not. The documents may come from teaching and research institutions in France or abroad, or from public or private research centers.
L'archive ouverte pluridisciplinaire HAL, est destinée au dépôt et à la diffusion de documents scientifiques de niveau recherche, publiés ou non, émanant des établissements d'enseignement et de recherche français ou étrangers, des laboratoires publics ou privés. 


\title{
Parametric instability in a rotating cylinder of gas subject to sinusoidal axial compression. Part 2. Weakly nonlinear theory
}

\author{
J.-P. R A CZ AND J. F. SCOTT \\ Laboratoire de Mécanique des Fluides et d'Acoustique, ECL, UCBL, INSA, CNRS, \\ 36 avenue Guy de Collongue, 69134 Ecully, France
}

(Received 26 February 2006 and in revised form 19 September 2007)

A weakly nonlinear analysis is presented of parametric instability in a rotating cylinder subject to periodic axial compression by small sinusoidal oscillations of one of its ends ('the piston'). Amplitude equations are derived for the pair of parametrically resonant (primary) inertial modes which were found to arise from linear instability in Part 1. These equations introduce an infinity of geostrophic mode amplitudes, representing a nonlinear modification of the mean flow, for which evolution equations are also derived. Consequences of the total system of equations are investigated for axisymmetric modes. Different possible outcomes are found at large times: (a) a fixed point, representing a saturated state in which the oscillatory toroidal vortices of the primary mode are phase-locked to the piston motion with half its frequency; (b) a limit cycle or chaotic attractor, corresponding to slow-time oscillations of the primary mode; or $(c)$ exponential divergence of the amplitudes to infinity. The latter outcome, a necessary condition for which is derived in the form of a threshold piston amplitude for divergence, invalidates the theory, inducing a gross change in the character of the flow and providing a route out of the weakly nonlinear regime. Non-zero fixed-point branches arise via bifurcations from both sides of the linear neutral curve, where the basic flow changes local stability. The loweramplitude branch is shown to be unstable, while the upper one may lose local stability, resulting in a Hopf bifurcation to a limit cycle, which can subsequently become aperiodic via a series of further bifurcations. Typically, during the resulting oscillations, whether periodic or not, the perturbation first grows from small amplitude owing to basic-flow instability, then nonlinear detuning of the parametric resonance causes decay back to small amplitude in the second half of the cycle, which then restarts.

\section{Introduction}

Growth of inertial waves due to externally imposed sinusoidal perturbations of rotating flow has frequently been observed. For instance, experiments were reported by Fultz (1959), McEwan (1970), Manasseh (1992) and others in the case of direct resonance, i.e. when the external forcing frequency is close to that of an inertial mode, whereas studies by Malkus (1989) and Eloy, Le Gal \& Le Dizés (2003) concern the elliptic instability, which can be interpreted theoretically (Waleffe 1990) as being due to a parametric inertial-wave resonance. The end result of direct resonance may lie in the linear regime if the forcing is sufficiently weak compared with the viscous damping which then limits modal growth, but otherwise nonlinearity intervenes, as 
is always the case for a parametric instability. McEwan (1970) and Malkus (1989) observed nonlinear effects, followed by wave breaking and transition to turbulence, whereas Eloy et al. (2003) found different parameter regimes in which the unstable mode either $(a)$ saturated at finite amplitude, $(b)$ underwent breaking and transition to turbulence, then relaminarized, leading to a periodic cycle of laminar and turbulent flows, or $(c)$ became turbulent and remained so.

To help explain his experimental results, McEwan (1970) proposed a theoretical model of a directly resonant inertial mode, which, though heuristic and relatively crude, contains many of the elements we will find from the detailed analysis of this paper for a different problem involving parametric instability. McEwan's model consists of a pair of differential equations for the time evolution of the amplitude and phase (relative to the external forcing) of the resonant mode. Nonlinear interaction of the oscillating mode with itself leads to mean forcing, hence modifying the mean flow (solid-body rotation in the absence of nonlinearity) as described by Busse (1968). This axisymmetric modification of the mean flow (modelled by McEwan as a single parameter representing the average change in the rotation rate), shifts the frequency of the mode a little, a shift which was later quantified in the perturbation analysis of Gunn \& Aldridge (1990). The resulting detuning of the resonance causes the modal phase relative to the external forcing to evolve, which in turn alters the growth of the modal amplitude, even producing modal decay if the phase becomes such that energy is extracted from the modal oscillations. As we shall see, our detailed asymptotic analysis leads to a similar physical picture of the effects of nonlinearity. The modification of the mean flow owing to nonlinearity appears in the analysis as an infinity of zero-frequency (geostrophic) modes, for which amplitude equations are derived in addition to those of the primary modes. It seems likely that the scenario described above is generic to problems involving resonant (direct or parametric) growth of primary modes in which there are zero-frequency (or nearly so) modes (the geostrophic ones in our case) which undergo nonlinear mean forcing by the primary mode and whose existence modifies the primary-mode frequency.

In the case of the elliptic instability, Waleffe (1989) undertook a weakly nonlinear analysis of a rotating, slightly elliptic, infinite cylinder of inviscid fluid. He obtained a set of evolution equations for the primary mode amplitude and the mean-flow correction induced by nonlinear forcing by the primary mode, in the manner discussed above. Although Waleffe's problem and ours are not the same, for instance the unstable mode is different, his amplitude equations have the same form as ours when viscosity is neglected. The equations are integrable and, depending upon the initial conditions, the solution is either periodic or goes from a fixed point back to the same point as time runs between $\pm \infty$. In particular, if the perturbation is infinitesimal to begin with, it grows from the linear regime owing to instability, but subsequently reaches a maximum and then decays back to zero. This unexpected (given the instability of the basic flow) behaviour is symptomatic of the singular nature of the inviscid problem, other aspects of which were discussed in Part 1 (Racz \& Scott 2008). Waleffe (1989) attempted to allow for viscosity by the expedient of adding linear damping terms to the inviscid amplitude equations, without carrying out the viscous analysis required to confirm such an approach. As we shall see, this misses a nonlinear term which requires viscosity for its existence and also does not allow for the infinity of geostrophic degrees of freedom which arise when volumetric viscous damping of the geostrophic flow is included. 
Again for the elliptic instability, the Appendix of Mason \& Kerswell (1999) gives a weakly nonlinear analysis of a finite cylinder allowing for viscosity, results of which are compared with their numerical work. Rather than deriving amplitude equations, they suppose from the start that the primary mode saturates to constant amplitude. This is equivalent to restricting attention to the fixed points of the amplitude equations and excludes other outcomes, such as a limit cycle, or divergence to infinity which, as we shall see, can also arise. Furthermore, the flow considered by Mason \& Kerswell in both their numerical and analytical studies satisfies theoretically convenient, but unrealistic, stress-free boundary conditions on the endwalls of the cylinder. As they recognize, no-slip conditions lead to boundary layers on the endwalls, which alters the problem significantly, changing not just the detailed values, but the order of magnitude of the geostrophic modal damping and mean forcing, quantities which are at the physical heart of the problem.

As in Part 1, the particular problem studied is that of flow inside a finite cylinder of radius $a$, rotating (along with its endwalls) at angular velocity $\Omega$ about its axis, one of whose ends (the piston) executes small sinusoidal oscillations of frequency $\omega_{0}$ and hence subjects the gas inside the cylinder to periodic compression. Linear instability was studied in Part 1, where it was found to take the form of growing pairs of inertial modes satisfying a parametric resonance conditions. In this paper, we allow for weak nonlinearity and derive coupled amplitude equations for the primary mode pair and the geostrophic modes which arise owing to nonlinearity, as discussed earlier. Section 2 introduces the asymptotic expansion of the mode amplitudes and gives some properties of the nonlinear interaction coefficients which are used later. For simplicity, and because we have fewer quantitative results for the non-axisymmetric case, attention is restricted to axisymmetric primary modes in the remainder of the paper (apart from Appendix C, which gives the amplitude equations for the non-axisymmetric case and briefly discusses some consequences). Section 3 derives the amplitude equations, and $\S 4$ examines their consequences. Throughout, the reader is assumed to be familiar with the formulation and notation of Part 1. References to equations in Part 1 take the form (I.n.nn), e.g. (I.3.9) refers to equation (3.9) of Part 1. Similar notation will be used to refer to sections and figures in Part 1. Appendices $\mathrm{D}$ to $\mathrm{H}$ give details of the calculations and are available as a supplement to the online version of the paper, or from the JFM Editorial Office.

Before proceeding further, although they were discussed in Part 1, geostrophic modes are especially important in this paper and it is perhaps best to recall their properties briefly. A geostrophic mode $\mu$ has zero frequency $\left(\omega^{(\mu)}=0\right.$ in the notation of Part 1) and the associated flow $\left(\boldsymbol{u}^{(\mu)}(\boldsymbol{X})\right)$ is two-dimensional, independent of the axial coordinate $Z$ (formally, the axial index $m_{\mu}=0$ ) and taking place in planes perpendicular to the axis, i.e. $u_{Z}^{(\mu)}=0$. Any such two-dimensional flow can be expressed as a linear combination of geostrophic modes and is steady according to linear inviscid theory without piston motion. The simplest geostrophic modes, and most important in the present paper, are axisymmetric (azimuthal order $n_{\mu}=0$ ). Their only nonzero velocity component is $u_{\theta}^{(\mu)}(r)$, corresponding to differential rotation of cylinders of fluid of constant $r$ about the $Z$-axis (recall figure I.2a). Although individual modes appear naturally in the analysis via their complex amplitudes, it is sometimes more enlightening to sum contributions from all geostrophic modes and refer to the geostrophic flow as a whole. 


\section{Asymptotic expansion and nonlinear interaction coefficients}

As in Part 1, the perturbation to the basic flow is written in terms of the basis set of inviscid inertial-mode eigenfunctions as

$$
\boldsymbol{u}=\sum_{\mu} B_{\mu}(t) \boldsymbol{u}^{(\mu)}(\boldsymbol{X})
$$

and the modal amplitudes evolve according to (I.3.9):

$$
\begin{aligned}
& \frac{\mathrm{d} B_{\mu}}{\mathrm{d} t}+\mathrm{i} \omega^{(\mu)} B_{\mu}=\underbrace{\frac{\mathrm{d}}{\mathrm{d} t}\left\{\left(1-\left(\frac{h}{h_{0}}\right)^{2}\right) \int u_{Z}^{(\mu)^{*}} u_{Z} \mathrm{~d}^{3} \boldsymbol{X}\right\}}_{\text {Piston motion }} \\
& +\operatorname{Re}^{-1}\{\underbrace{\int_{r=1}\left(\boldsymbol{u}_{\perp}^{(\mu)^{*}}+\left(\frac{h}{h_{0}}\right)^{2} \boldsymbol{e}_{Z} u_{Z}^{(\mu)^{*}}\right) \cdot(\boldsymbol{n} \cdot \nabla \boldsymbol{u}) \mathrm{d}^{2} \boldsymbol{X}}_{\text {Sidewall viscous term }} \\
& +\underbrace{\int_{Z=0, h_{0}}\left(\left(\frac{h_{0}}{h}\right)^{2} \boldsymbol{u}_{\perp}^{(\mu)^{*}}+\boldsymbol{e}_{Z} u_{Z}^{(\mu)^{*}}\right) \cdot(\boldsymbol{n} \cdot \nabla \boldsymbol{u}) \mathrm{d}^{2} \boldsymbol{X}}_{\text {Endwall viscous term }} \\
& +\underbrace{\int \boldsymbol{u} \cdot \mathscr{D}\left(\boldsymbol{u}_{\perp}^{(\mu)^{*}}+\left(\frac{h}{h_{0}}\right)^{2} \boldsymbol{e}_{Z} u_{Z}^{(\mu)^{*}}\right) \mathrm{d}^{3} \boldsymbol{X}}_{\text {Volumetric viscous term }}\} \\
& +\int[\underbrace{\boldsymbol{u} \cdot(\boldsymbol{u} \cdot \nabla)}_{\text {Nonlinearity }}+\underbrace{\boldsymbol{U} \cdot(\boldsymbol{u} \cdot \nabla)+\boldsymbol{u} \cdot(\boldsymbol{U} \cdot \nabla)}_{\text {Effects of viscosity on the basic flow }}] \\
& \times\left(\boldsymbol{u}_{\perp}^{(\mu)^{*}}+\left(\frac{h}{h_{0}}\right)^{2} \boldsymbol{e}_{Z} u_{Z}^{(\mu)^{*}}\right) \mathrm{d}^{3} \boldsymbol{X} .
\end{aligned}
$$

Consider an unstable pair of modes, $\mu_{+}, \mu_{-}$, growing from the linear regime until it is affected by nonlinearity. For reasons that will become apparent later, nonlinearity intervenes when the amplitude of the primary modes reaches $O\left(\varepsilon^{1 / 2}\right)$ (recall from Part 1 that $\varepsilon$ is a small parameter representing the amplitude of piston oscillations and on which the asymptotic analysis is based), hence the expansion of the modal amplitudes in powers of $\varepsilon^{1 / 2}$ :

$$
B_{\mu}=\varepsilon^{1 / 2} B_{\mu}^{[1]}+\varepsilon B_{\mu}^{[2]}+\varepsilon^{3 / 2} B_{\mu}^{[3]}+\cdots,
$$

where the leading-order term contains only the primary modes, i.e. $B_{\mu}^{[1]}=0$ unless $\mu=\mu_{+}$or $\mu=\mu_{-}$(and their conjugates in the non-axisymmetric case). Equation (2.3) is used in the exact amplitude evolution equation (2.2) and successive powers of $\varepsilon^{1 / 2}$ examined. At leading order, effects of piston-motion, viscosity and nonlinearity, represented by the right-hand side of (2.2), do not contribute and so

$$
B_{\mu}^{[1]}=A_{\mu}(T) \exp \left(-\mathrm{i} \omega^{(\mu)} t\right)
$$

expresses oscillations of the primary modes at their natural inviscid frequencies, with amplitudes $A_{\mu_{ \pm}}=A_{ \pm}(T)$ which are functions of the slow time $T=\varepsilon t$. 
Evolution equations for the $A_{ \pm}$are obtained, as usual, by elimination of secularity at higher order. As we shall see, no secularities occur at $O(\varepsilon)$ (which is the reason why the perturbation amplitude can grow to $O\left(\varepsilon^{1 / 2}\right)$, rather than $O(\varepsilon)$, before nonlinearity becomes significant) and the amplitude equations arise at $O\left(\varepsilon^{3 / 2}\right)$. The right-hand side of (2.2) contributes at these orders and its asymptotic expansion is then required. The linear terms on the right-hand side of (2.2) have already been treated in Part 1 and it remains to express the nonlinear term. Using (2.1), (2.3) and $h / h_{0}=1+O(\varepsilon)$, this term has the expansion

$$
\begin{aligned}
\int \boldsymbol{u} \cdot(\boldsymbol{u} \cdot \nabla)\left(\boldsymbol{u}_{\perp}^{(\mu)^{*}}+\left(\frac{h}{h_{0}}\right)^{2} \boldsymbol{e}_{Z} u_{Z}^{(\mu)^{*}}\right) \mathrm{d}^{3} \boldsymbol{X} & =\mathrm{i} \varepsilon \sum_{v_{1} v_{2}} \Lambda_{\mu v_{1} v_{2}} B_{v_{1}}^{[1]} B_{v_{2}}^{[1]} \\
& +2 \mathrm{i} \varepsilon^{3 / 2} \sum_{v_{1} v_{2}} \Lambda_{\mu v_{1} v_{2}} B_{v_{1}}^{[1]} B_{v_{2}}^{[2]}+\cdots,
\end{aligned}
$$

which provides the nonlinear contributions at $O(\varepsilon)$ and $O\left(\varepsilon^{3 / 2}\right)$, where

$$
\Lambda_{\mu \nu_{1} \nu_{2}}=-\frac{1}{2} \mathrm{i} \int\left(u_{\mathrm{i}}^{\left(\nu_{1}\right)} u_{j}^{\left(\nu_{2}\right)}+u_{i}^{\left(\nu_{2}\right)} u_{j}^{\left(\nu_{1}\right)}\right) \frac{\partial u_{\mathrm{i}}^{(\mu) *}}{\partial X_{j}} \mathrm{~d}^{3} \boldsymbol{X},
$$

with a summation convention over repeated indices, defines a set of nonlinear interaction coefficients which play an important role in the present paper. Note that $\Lambda_{\mu v_{1} v_{2}}$ is symmetric with respect to its last two indices.

$\Lambda_{\mu v_{1} \nu_{2}}$ can be expressed in terms of integrals of products of Bessel functions (see Appendix D for details). In the process, it is found to be real and zero unless

$$
n_{\mu}=n_{v_{1}}+n_{v_{2}}, \quad m_{\mu}= \pm m_{v_{1}} \pm m_{v_{2}},
$$

there being four choices of sign in the second condition. These conditions constrain possible nonlinear interactions. Another useful property of the $\Lambda_{\mu v_{1} \nu_{2}}$ (also derived in Appendix D) is

$$
\Lambda_{\mu \nu v^{*}}=0, \quad m_{\mu}=0
$$

for any geostrophic mode $\mu$ (i.e. $m_{\mu}=0$, as indicated explicitly in (2.8)) and where, as in Part 1, $v^{*}$ denotes the modal conjugate of $v$. A result equivalent to (2.8) was derived by Greenspan (1969) for a general container. As we shall see in later analysis, (2.8) disallows a number of nonlinear interactions involving geostrophic modes which would otherwise significantly modify the dynamics. Note that, like the linear coupling matrices $C_{\mu \nu}$ and $D_{\mu \nu}$ introduced in Part 1, the $\Lambda_{\mu \nu_{1} \nu_{2}}$ depend only on the cylinder aspect ratio $h_{0}$.

\section{Amplitude equations for axisymmetric primary modes}

From here on, we restrict attention to the axisymmetric case. As we saw in Part 1, the pair then consists of conjugate modes $\mu_{+}$and $\mu_{-}=\mu_{+}^{*}$ having the same nonzero axial order $m_{+}$, frequencies $\omega_{-}=-\omega_{+}$which satisfy the resonance condition $2 \omega_{+}=\omega_{+}-\omega_{-}=\omega_{0}+O(\varepsilon)$, and leading-order amplitudes related by $A_{-}=A_{+}^{*}$. 


\subsection{Order $\varepsilon$}

At $O(\varepsilon)$, only the nonlinear term contributes to the right-hand side of (2.2) (the linear terms are $\left.O\left(\varepsilon^{3 / 2}\right)\right)$ and, using (2.4) and (2.5), we have

$$
\frac{\partial B_{\mu}^{[2]}}{\partial t}+\mathrm{i} \omega^{(\mu)} B_{\mu}^{[2]}=\mathrm{i} \sum_{\substack{\nu_{1}, v_{2}=\\ \mu_{+}, \mu_{+}^{*}}} \Lambda_{\mu v_{1} \nu_{2}} A_{v_{1}} A_{\nu_{2}} \exp -\left(\mathrm{i}\left(\omega^{\left(\nu_{1}\right)}+\omega^{\left(\nu_{2}\right)}\right) t\right),
$$

where the notation indicates that the sum, representing $O(\varepsilon)$ forcing due to quadratic interactions between the primary modes, is to be taken by letting $v_{1}$ and $v_{2}$ run over $\mu_{+}$and $\mu_{+}^{*}$. On the other hand, the mode $\mu$, whose $O(\varepsilon)$ amplitude evolves according to (3.1), is not restricted to be one of the primary modes. The four terms in the sum of (3.1) are of two types: those with $v_{1}=v_{2}$ have frequencies $\pm 2 \omega_{+}$(second harmonic), while those with $v_{1}=v_{2}^{*}$ are of zero frequency (steady on the fast time scale). The coefficient $\Lambda_{\mu v_{1} \nu_{2}}=0$ unless (2.7) is satisfied, so only the two families $n_{\mu}=m_{\mu}=0$ and $n_{\mu}=0, m_{\mu}=2 m_{+}$are subject to forcing.

The solution of (3.1) is

$$
B_{\mu}^{[2]}=\mathscr{A}_{\mu}(T) \exp \left(-\mathrm{i} \omega^{(\mu)} t\right)+\sum_{\substack{v_{1}, \nu_{2}=\\ \mu_{+}, \mu_{+}^{*}}} \frac{\Lambda_{\mu \nu_{1} \nu_{2}} A_{\nu_{1}} A_{\nu_{2}}}{\omega^{(\mu)}-\omega^{\left(\nu_{1}\right)}-\omega^{\left(\nu_{2}\right)}} \exp \left(-\mathrm{i}\left(\omega^{\left(\nu_{1}\right)}+\omega^{\left(\nu_{2}\right)}\right) t\right),
$$

where $\mathscr{A}_{\mu}$ is the next-order correction to the leading-order slowly varying amplitude $A_{\mu}$ and the sum is zero unless $n_{\mu}=m_{\mu}=0$ or $n_{\mu}=0, m_{\mu}=2 m_{+}$. If $m_{\mu}=0, \omega^{(\mu)}=0$ and there is an apparent division by zero in (3.2) when $v_{1}=v_{2}^{*}$, reflecting a potential secularity of the solution of (3.1). However, according to (2.8), the corresponding forcing terms in (3.1) are in fact zero (i.e. there is no mean forcing of geostrophic modes at this order, a result obtained by Greenspan (1969) for a general container in the absence of viscosity and piston motion) and the secularity that would otherwise occur is avoided. In consequence, terms with $v_{1}=v_{2}^{*}$ should be dropped from the sum in (3.2) when $m_{\mu}=0$ and their absence taken into account when interpreting later equations which inherit the apparent divisions by zero. As noted earlier, lack of secularity at this order is the reason why the mode pair grows to amplitude $O\left(\varepsilon^{1 / 2}\right)$ before nonlinearity makes itself felt.

Whereas (3.2) represents a small correction for the primary modes, because they are already present at leading order, it is asymptotically dominant for all other modes. According to (3.2), the $O(\varepsilon)$ flow is a superposition of free inertial-mode oscillations with amplitudes $\mathscr{A}_{\mu}$ and the response to quadratic forcing of modes in the families $n_{\mu}=m_{\mu}=0$ and $n_{\mu}=0, m_{\mu}=2 m_{+}$. For geostrophic $\mu, \omega^{(\mu)}=0$ and the first term in (3.2) is steady on the fast time scale, representing an $O(\varepsilon)$ modification of the mean flow with the potential to affect the primary mode dynamics as in Gunn \& Aldridge (1990). As we shall see shortly, the $\mathscr{A}_{\mu}$ of the particular geostrophic family $n_{\mu}=m_{\mu}=0$ (axisymmetric, as well as geostrophic) do indeed appear in the amplitude equations of the primary modes. In consequence, modes with $n_{\mu}=m_{\mu}=0$ play an important role in this paper. Note that, although the first term in (3.2) appears as unforced here, amplitude equations for the $\mathscr{A}_{\mu}$ (derived in $\S 3.3$ for geostrophic $\mu$ ) arising at $O\left(\varepsilon^{2}\right)$ show that it is, in fact, the consequence of nonlinear forcing of $O\left(\varepsilon^{2}\right)$ acting over long times of $O\left(\varepsilon^{-1}\right)$. 
At this order, (2.2) gives

$$
\frac{\partial B_{\mu}^{[3]}}{\partial t}+\mathrm{i} \omega^{(\mu)} B_{\mu}^{[3]}=\Psi_{\mu}^{L}+\Psi_{\mu}^{N L}-\frac{\mathrm{d} A_{\mu}}{\mathrm{d} T} \exp \left(-i \omega^{(\mu)} t\right)
$$

where the final term comes from taking the time derivative of (2.4), while $\Psi_{\mu}^{L}$ and $\Psi_{\mu}^{N L}$ arise, respectively, from the linear and nonlinear terms on the right-hand side of (2.2). The linear terms were treated in $\S 4.1$ of Part 1 and the results carry over directly. Thus, $\Psi_{\mu}^{L}$ is the sum of the right-hand sides of (I.4.5)-(I.4.7), divided by $\delta \varepsilon$. As regards $\Psi_{\mu}^{N L}$, using (3.2) in (2.5), we obtain

$$
\begin{aligned}
\Psi_{\mu}^{N L}=2 \mathrm{i} \sum_{\substack{\lambda \\
\nu=\mu_{+}, \mu_{+}^{*}}} \Lambda_{\mu \nu \lambda} A_{\nu} \mathscr{A}_{\lambda} \exp \left(-\mathrm{i}\left(\omega^{(\nu)}+\omega^{(\lambda)}\right) t\right)+\frac{1}{2} \mathrm{i} \\
\sum_{\substack{\nu_{1}, \nu_{2}, \nu_{3}=\\
\mu_{+}, \mu_{+}^{*}}} F_{\mu \nu_{1} \nu_{2} \nu_{3}} A_{\nu_{1}} A_{\nu_{2}} A_{\nu_{3}} \exp \left(-\mathrm{i}\left(\omega^{\left(\nu_{1}\right)}+\omega^{\left(\nu_{2}\right)}+\omega^{\left(\nu_{3}\right)}\right) t\right),
\end{aligned}
$$

where

$$
F_{\mu \nu_{1} \nu_{2} v_{3}}=4 \sum_{\lambda} \frac{\Lambda_{\lambda v_{1} \nu_{2}} \Lambda_{\mu v_{3} \lambda}}{\omega^{(\lambda)}-\omega^{\left(\nu_{1}\right)}-\omega^{\left(\nu_{2}\right)}}
$$

is a set of real cubic-interaction coefficients and the notation $\lambda$ has been used in (3.4) and (3.5) to emphasize that the sum over $\lambda$ includes all modes, whereas the $v$ are restricted to the mode pair $\mu_{+}, \mu_{+}^{*}$. Both $\Psi_{\mu}^{L}$ and $\Psi_{\mu}^{N L}$, occurring in (3.3), consist of a sum of oscillatory exponentials in $t$ and we now look for resonant terms (i.e. those with frequency $\left.\omega_{\mu}+O(\varepsilon)\right)$ to derive the amplitude equations of the primary modes as non-secularity conditions for $\mu=\mu_{+}$and $\mu=\mu_{+}^{*}$. That is, the sum of all resonant terms should be zero, otherwise the solution of (3.3) will be secular.

Resonant terms in $\Psi_{\mu}^{L}$ were identified in Part 1, leading to the linear amplitude equations (I.4.12) and (I.4.13). Here, the results of Part 1 are simply carried over into the nonlinear amplitude equation below. As regards $\Psi_{\mu}^{N L}$ with $\mu=\mu_{+}$, three terms in the second sum of (3.4), namely $v_{1}=v_{2}=\mu_{+}$and $v_{3}=\mu_{+}^{*}$ and the two permutations of these values, contribute the resonant term $\mathrm{i} G\left|A_{+}\right|^{2} A_{+} e^{-\mathrm{i} \omega_{+} t}$, where

$$
G=\frac{1}{2} F_{\mu_{+} \mu_{+} \mu_{+} \mu_{+}^{*}}+F_{\mu_{+} \mu_{+} \mu_{+}^{*} \mu_{+}},
$$

and we have used $F_{\mu \nu_{1} \nu_{2} \nu_{3}}=F_{\mu \nu_{2} \nu_{1} \nu_{3}}$ (which follows from (3.5) and symmetry of $\Lambda$ with respect to its last two indices). The first sum in (3.4) with $\mu=\mu_{+}$yields resonance when $v=\mu_{+}$and $\lambda$ is a mode with $\omega^{(\lambda)}=0$, i.e. $\lambda$ is geostrophic $\left(m_{\lambda}=0\right)$. Since, according to (2.7), $\Lambda_{\mu_{+} \mu_{+} \lambda}=0$ unless $n_{\lambda}=0$, we obtain the resonant contribution $2 \mathrm{i} A_{+} \exp \left(-\mathrm{i} \omega_{+} t\right) \sum_{\sigma \in M} \Lambda_{\mu_{+} \mu_{+} \sigma} \mathscr{A}_{\sigma}$, where $M$ denotes the family of axisymmetric, geostrophic modes, $n_{\sigma}=m_{\sigma}=0$. Collecting together the linear resonant terms identified in Part 1 and the non-linear ones just described, the non-secularity condition for $\mu=\mu_{+}$gives the primary-mode amplitude equation

$$
\frac{\mathrm{d} A_{+}}{\mathrm{d} T}=\mathrm{i} \omega_{+} C \mathrm{e}^{-\mathrm{i} \Delta T} A_{+}^{*}+\left(2 \mathrm{i} \sum_{\sigma \in M} \Lambda_{\mu_{+} \mu_{+} \sigma} \mathscr{A}_{\sigma}-\varepsilon^{-1} \overline{R e}-1 / 2 d_{+}\right) A_{+}+\mathrm{i} G\left|A_{+}\right|^{2} A_{+},
$$

where, among the notation inherited from Part 1, $C=C_{\mu_{+} \mu_{+}^{*}}<0$ is the piston-motion coupling coefficient of the mode pair, $\Delta=\varepsilon^{-1}\left(\omega_{0}-2 \omega_{+}\right)$is a detuning parameter 
expressing the scaled departure of the piston frequency from parametric resonance, $\overline{R e}$ is the Reynolds number (of order $\varepsilon^{2}$, as in Part 1), and $d_{+}$is the complex modal damping coefficient given by $d_{+}=d_{\mu_{+}}$and (I.4.10). Similar reasoning applied to (3.3) with $\mu=\mu_{+}^{*}$ yields the conjugate of (3.7). In writing (3.7), we suppose there are no secular terms other than those identified above. Equation (3.7) and its conjugate are nonlinear generalizations of the linear mode-pair equations of Part 1 . Note that, since $M$ represents modes with $n=m=0$, which are real, the amplitudes $\mathscr{A}_{\sigma}$ in (3.7) are also real, as are the coefficients $\omega_{+} C, \Lambda_{\mu_{+} \mu_{+} \sigma}$ and $G$. Expressions for the nonlinear coefficients $\Lambda_{\mu_{+} \mu_{+} \sigma}$ and $G$ can be found in Appendix A.

The linear terms on the right-hand side of (3.7) express the piston-motion and viscous effects already present in Part 1 . The sum over $\mathscr{A}_{\sigma}$ arises from the first term in (3.2) and represents the modification of the primary-mode dynamics by axisymmetric geostrophic modes discussed earlier. Since the coefficient $2 \mathrm{i} \sum_{\sigma \in M} \Lambda_{\mu_{+} \mu_{+} \sigma} \mathscr{A}_{\sigma}$ is purely imaginary, it can be thought of as inducing a small shift in the frequency of the primary mode equal to $-2 \varepsilon \sum_{\sigma \in M} \Lambda_{\mu_{+} \mu_{+} \sigma} \mathscr{A}_{\sigma}$. Note that non-axisymmetric, geostrophic modes $\lambda$ have been excluded by $\Lambda_{\mu_{+} \mu_{+} \lambda}=0$, i.e. they do not have the correct spatial structure to interact with the primary mode. The final term in (3.7) originates from the sum in (3.2) and corresponds to cubic interactions of the primary mode with itself. It has the familiar Landau form with an imaginary Landau constant, i $G$. Being imaginary, this term can also be interpreted as a nonlinear correction to the primary-mode frequency, this time owing to the primary mode itself.

\subsection{Order $\varepsilon^{2}$}

Since the geostrophic mode amplitudes appear in the primary-mode equation (3.7), we must go to $O\left(\varepsilon^{2}\right)$. This requires more precise asymptotics than we have required so far. To this end, let $\sigma$ be a geostrophic mode (not necessarily axisymmetric), then (2.2) leads to the exact (no asymptotics) equation

$$
\begin{aligned}
\frac{\mathrm{d} B_{\sigma}}{\mathrm{d} t}+R e^{-1} K^{(\sigma) 2} B_{\sigma}= & R e^{-1}\left(\frac{h_{0}}{h}\right)^{2} \int_{Z=0, h_{0}} \boldsymbol{u}^{(\sigma)^{*}} \cdot(\boldsymbol{n} \cdot \nabla \boldsymbol{u}) \mathrm{d}^{2} \boldsymbol{X} \\
& +\int[\boldsymbol{u} \cdot(\boldsymbol{u} \cdot \nabla)+\boldsymbol{U} \cdot(\boldsymbol{u} \cdot \nabla)+\boldsymbol{u} \cdot(\boldsymbol{U} \cdot \nabla)] \boldsymbol{u}^{(\sigma)^{*}} \mathrm{~d}^{3} \boldsymbol{X}
\end{aligned}
$$

where, in deriving this result from (2.2), the following properties of geostrophic modes have been used: $\omega^{(\sigma)}=0, u_{Z}^{(\sigma)}=0, \boldsymbol{u}^{(\sigma)}$ is independent of $Z$, and $\boldsymbol{u}^{(\sigma)}=0$ on $r=1$ (recall that satisfaction of no-slip on the sidewall is specific to the particular choice of geostrophic modes made in Part 1; that choice becomes significant here). The volumetric viscous term (appearing on the left-hand side of (3.8)) has also been rewritten using (I.2.12), (I.A.8), (I.2.17), the divergence theorem and the boundary conditions on $\boldsymbol{u}$.

As a first step in the asymptotic analysis, (3.8) is first approximated, correct to $O\left(\varepsilon^{2}\right)$, by replacing $R e$ with its mean value $\overline{R e}$ and $h_{0} / h$ with 1 . In principle, the amplitude equations could then be derived by imposing non-secularity at $O\left(\varepsilon^{2}\right)$, but it is simpler to use an equivalent fast-time averaging approach. $B_{\sigma}$ is regarded as a function of both the fast and slow times, $t$ and $T$, and averaging with respect to $t$ applied to (3.8), approximated as described above. The time derivative is expressed using

$$
\left\langle\frac{\mathrm{d} B_{\sigma}}{\mathrm{d} t}\right\rangle=\left\langle\frac{\partial B_{\sigma}}{\partial t}+\varepsilon \frac{\partial B_{\sigma}}{\partial T}\right\rangle=\varepsilon \frac{\partial\left\langle B_{\sigma}\right\rangle}{\partial T},
$$


where \langle\rangle denotes fast-time averaging and we have used the facts that: (i) the fasttime average of a fast-time derivative is zero (an expression of non-secularity); and (ii) fast-time averaging and slow-time differentiation commute. Taking the fast-time average of (3.2) with $\mu=\sigma$ (and recalling that the $v_{1}=v_{2}^{*}$ terms in the sum of (3.2), which have zero frequency and would thus appear to contribute to the average, should be dropped, as discussed following (3.2)) gives $\left\langle B_{\sigma}\right\rangle=\varepsilon \mathscr{A}_{\sigma}(T)$, correct to $O(\varepsilon)$. Thus, we obtain

$$
\begin{aligned}
\varepsilon^{2}\left(\frac{\mathrm{d} \mathscr{A}_{\sigma}}{\mathrm{d} T}+\varepsilon^{-1} \overline{R e}^{-1} K^{(\sigma)^{2}} \mathscr{A}_{\sigma}\right)=\overline{R e}^{-1} \int_{Z=0, h_{0}} \boldsymbol{u}^{(\sigma)^{*}} \cdot(\mathbf{n} \cdot \nabla\langle\boldsymbol{u}\rangle) \mathrm{d}^{2} \boldsymbol{X} \\
+\int\left\langle[\boldsymbol{u} \cdot(\boldsymbol{u} \cdot \nabla)+\boldsymbol{U} \cdot(\boldsymbol{u} \cdot \nabla)+\boldsymbol{u} \cdot(\boldsymbol{U} \cdot \nabla)] \boldsymbol{u}^{(\sigma)^{*}}\right\rangle \mathrm{d}^{3} \boldsymbol{X}
\end{aligned}
$$

correct to $O\left(\varepsilon^{2}\right)$, and we next show that the terms involving $\boldsymbol{U}$ can be neglected.

Since $\boldsymbol{U}$ arises from the basic flow, it is periodic with the piston period and can be expressed as a Fourier series containing frequencies which are multiples of $\omega_{0}$. The corresponding fast-time frequencies are multiples of $2 \omega_{+}$, thanks to the parametric resonance condition $\omega_{0}=2 \omega_{+}+O(\varepsilon)$. On the other hand, the first term in the expansion of $\boldsymbol{u}$ has fast-time frequency $\omega_{+}$. As a result, the fast-time average of the product of $\boldsymbol{U}$ and the leading-order term in $\boldsymbol{u}$ is zero and we must proceed to $O(\varepsilon)$ in the asymptotic expansion of $\boldsymbol{u}$ before obtaining a non-zero contribution to the terms involving $\boldsymbol{U}$ in (3.10). As we saw in Part 1 (in the paragraph following (I.4.7)), $\boldsymbol{U}$ is $O(\varepsilon)$ within the sidewall boundary layer of thickness $O(\varepsilon)$, outside which it is small compared with $\varepsilon$ provided basic-flow resonances are excluded, as we did in Part 1 and continue to do here. Thus, estimating the order of magnitude of the terms in (3.10) involving $\boldsymbol{U}$, they are found to be small compared with $\varepsilon^{2}$ and hence negligible to the order to which we are working. Dropping these terms, (3.10) becomes

$$
\begin{aligned}
& \frac{\mathrm{d} \mathscr{A}_{\sigma}}{\mathrm{d} T}+\varepsilon^{-1} \overline{R e}^{-1} K^{(\sigma)^{2}} \mathscr{A}_{\sigma} \\
& =\varepsilon^{-2}\left\{\overline{\operatorname{Re}}^{-1} \int_{Z=0, h_{0}} \boldsymbol{u}^{(\sigma)^{*}} \cdot(\boldsymbol{n} \cdot \nabla\langle\boldsymbol{u}\rangle) \mathrm{d}^{2} \boldsymbol{X}+\int\left\langle\boldsymbol{u} \cdot(\boldsymbol{u} \cdot \nabla) \boldsymbol{u}^{(\sigma)^{*}}\right\rangle \mathrm{d}^{3} \boldsymbol{X}\right\}
\end{aligned}
$$

the embryo of an amplitude equation for geostrophic modes. The integrals on the right-hand side of (3.11) can be understood as forcing of the given mode by: $(a)$ the mean viscous stress at the end walls, and $(b)$ the mean momentum flux (analogous to the Reynolds stress in turbulent flows) throughout the cylinder.

Asymptotic evaluation of the integrals on the right-hand side of (3.11) requires lengthy analysis, outlined in Appendix B and detailed in the online Appendices D-H. In the general case in which the geostrophic flow is non-axisymmetric, the result is the amplitude equation (B 12), in which the different terms on the right-hand side represent: (i) viscous friction, both volumetric and at the ends of the cylinder, tending to slow the geostrophic motion; (ii) nonlinear coupling between geostrophic modes; and (iii) nonlinear forcing of the geostrophic flow by the primary mode. Because the forcing is axisymmetric, if the geostrophic flow is initially axisymmetric, it remains so. Leaving aside the possibility of a secondary symmetry-breaking instability (which would lead to spontaneous growth of non-axisymmetric geostrophic modes and require the full equation (B 12)), we specialize to the axisymmetric case in what follows. The result is the geostrophic amplitude equation 


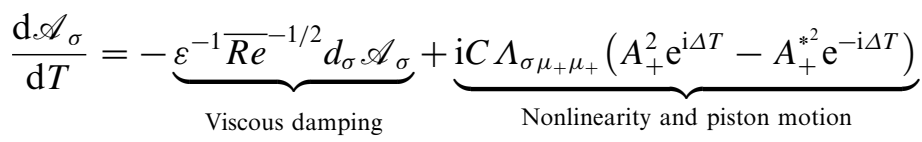

$$
\begin{aligned}
& +\underbrace{\varepsilon^{-1} \overline{R e}^{-1 / 2} \Gamma_{\sigma}\left|A_{+}\right|^{2}}_{\begin{array}{c}
\text { Nonlinearity and } \\
\text { viscosity }
\end{array}} \quad(\sigma \in M),
\end{aligned}
$$

in which coupling between geostrophic modes has now disappeared. As indicated by the annotation, the first term on the right-hand side expresses viscous friction, while the remainder represents nonlinear forcing, catalysed by piston motion and viscosity.

Equations (3.7) and (3.12) provide the evolution equations for the primary and axisymmetric geostrophic amplitudes used in the next section. The geostrophic amplitudes $\mathscr{A}_{\sigma}$ described by (3.12) are real, as are the coefficients $d_{\sigma}, C \Lambda_{\sigma \mu_{+} \mu_{+}}$ and $\Gamma_{\sigma}$. Expressions for $\Lambda_{\sigma \mu_{+} \mu_{+}}$and $\Gamma_{\sigma}$ can be found in Appendix A.

\section{Analysis and discussion}

Equations (3.7) and (3.12) form an infinite set of evolution equations for the primary and geostrophic mode amplitudes, $A_{+}$and $\mathscr{A}_{\sigma}$. Transforming to the new amplitude variable

$$
a=A_{+} \mathrm{e}^{\mathrm{i} \Delta T / 2}
$$

for the primary mode, leads to

$$
\begin{aligned}
& \frac{\mathrm{d} a}{\mathrm{~d} T}=\underbrace{\mathrm{i} \omega_{+} C a^{*}}_{\text {Piston motion }}+(\underbrace{\frac{1}{\mathrm{i}} \hat{\Delta}}_{\text {Detuning }}-\underbrace{\varepsilon^{-1} \overline{R e}-1 / 2}_{\begin{array}{c}
\text { Viscous } \\
\text { damping }
\end{array}} d_{+}^{r}+\underbrace{2 \mathrm{i} \sum_{\sigma \in M} \Lambda_{\mu_{+} \mu_{+} \sigma} \mathscr{A}_{\sigma}}_{\text {Geostrophic flow }}) a+\underbrace{\mathrm{i} G|a|^{2} a}_{\text {Cubic interactions }},
\end{aligned}
$$

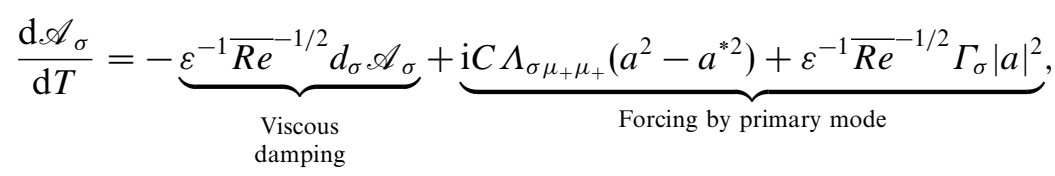

in which there are now no time-varying coefficients. Here, we have written $d_{+}^{r}$ for the real part of $d_{+}$, i.e. $d_{+}^{r}$ is the viscous damping factor of the primary mode, and

$$
\hat{\Delta}=\Delta-2 \varepsilon^{-1} \overline{R e}^{-1 / 2} d_{+}^{i}=\varepsilon^{-1}\left(\omega_{0}-2\left(\omega_{+}+\overline{R e}^{-1 / 2} D_{\mu_{+} \mu_{+}}^{i}\right)\right)
$$

is a detuning parameter expressing the scaled departure of the piston frequency from the viscous resonance condition $\omega_{0}=\omega_{c}=2\left(\omega_{+}+\overline{R e}^{-1 / 2} D_{\mu_{+} \mu_{+}}^{i}\right), D_{\mu_{+} \mu_{+}}^{i}$ being the imaginary part of $D_{\mu_{+} \mu_{+}}$, representing a small viscous correction to the inviscid modal frequency $\omega_{+}$. The appearance of $\hat{\Delta}$ in (4.2) is the only way in which the piston frequency enters the problem. We shall refer to solutions of (4.2), (4.3) which are independent of time (i.e. yielding zero for the right-hand sides) as fixed points.

The first term on the right-hand side of (4.2) results from the piston oscillations and is necessary for instability, while $d_{+}^{r}$ expresses viscous damping of the primary mode, both volumetric and due to the boundary layers. The final term in (4.2) arises from 
nonlinear interactions of the primary mode with itself and has the familiar Landau form. Note that the Landau coefficient, $\mathrm{i} G$, is purely imaginary, thus conserving energy. As discussed earlier, both the sum over the $\mathscr{A}_{\sigma}$ and the cubic term can be thought of as representing a small, $O(\varepsilon)$, shift in the frequency of the primary mode due to the leading-order geostrophic flow, $\boldsymbol{u}^{g}=\varepsilon \sum_{\sigma \in M} \mathscr{A}_{\sigma} \boldsymbol{u}^{(\sigma)}$, and interactions of the primary mode with itself. Since $u_{r}^{g}=u_{Z}^{g}=0$ and $\boldsymbol{u}^{g}$ is independent of $Z$, the geostrophic flow, $u_{\theta}^{g}(r)$, can be visualized as the steady (on the fast time scale) rotation of cylinders of fluid of constant $r$ about the cylinder axis, with a rotation rate which varies with $r$. This motion produces a small $(O(\varepsilon))$ modification of the basic solid-body rotation of the fluid in an inertial frame of reference, the resulting small shift in the primary-mode frequencies being sufficient that the parametric resonance, itself narrowband, is affected.

Turning to (4.3), the first term on the right-hand side represents the viscous damping of the geostrophic flow described above, while the remaining two terms express nonlinear forcing of that flow by the primary mode. The viscous damping coefficient is given by (I.4.10), in which the volumetric term represents friction between the differentially rotating cylinders of fluid which comprise the geostrophic flow. The other term in (I.4.10) corresponds to viscous friction at the endwalls, tending to slow the rotating cylinders. Since, according to (I.B.7), $D_{\sigma \sigma}=2^{1 / 2} / h_{0}$, all modes of the family $M$ would have the same damping rate in the absence of the volumetric term. Thus, in the absence of both forcing by the primary mode and volumetric damping, the geostrophic flow would decay exponentially owing to endwall friction, but maintain the same form of velocity profile $u_{\theta}^{g}(r)$ at all times. Primary-mode forcing is responsible for generating and maintaining the geostrophic flow and is resisted by the viscous effects just described. As is apparent from (4.3), there are two types of nonlinear forcing. The first, represented by the second term on the right-hand side, involves a combination of piston motion and nonlinearity, while the other requires viscosity. Note that, in the absence of piston motion and viscosity, there is no forcing by the primary mode, so the geostrophic flow does not arise. This is an extension of the results of Greenspan (1969) to higher order in $\varepsilon$ for the case of the cylinder.

If the geostrophic sum is dropped from (4.2), we obtain a generalized Landau equation for $a$. Amplitude equations of this form have been obtained for other problems involving parametric instability, for instance, the Faraday surface-wave instability (see e.g. Douady 1990, equation (7a) or Milner 1991, equation (22), without the spatial derivatives, corrected in detail by Miles 1993, Appendix D). It can be shown that solutions of the generalized Landau equation obtained by neglecting the geostrophic sum always approach a fixed point as $T \rightarrow \infty$, whereas, as we shall see, the full system of equations, (4.2), (4.3), has much richer large-time dynamics. Thus, it is important to allow for the effect of the geostrophic modes on the primary mode.

As noted above, if we neglect the volumetric damping term in (I.4.10), all the $d_{\sigma}$ coincide. In that case, the amplitude equations for modes of the family $M$ can be combined by multiplying (4.3) by $\Lambda_{\mu_{+} \mu_{+} \sigma}$ and summing over $\sigma$. This leads to amplitude equations for $a$ and $\mathscr{A}=\sum_{\sigma \in M} \Lambda_{\mu_{+} \mu_{+} \sigma \mathscr{A}_{\sigma}}$, i.e. the infinite system (4.2) and (4.3) is reduced to a finite one. Although obviously simpler than (4.2), (4.3), we know of no analytical solutions of either system of equations (other than the fixed points discussed below) and so no real advantage is gained by using the simpler system, since numerical solution is required in any case. Allowing for volumetric damping of the geostrophic modes should yield a more accurate description of high-order ones, so we keep the volumetric damping term in (4.3) in what follows. 
The primary-mode amplitude can be expressed using a real amplitude and phase as

$$
a=|a| \exp \left[-\frac{1}{2} \mathrm{i}\left(\psi+\frac{1}{2} \pi\right)\right]
$$

where $\psi$ is a phase angle. Recalling that the scaling $\boldsymbol{u}=O\left(\varepsilon^{1 / 2}\right)$ means that the perturbation dominates the flow, the leading-order velocity field can be written in terms of $|a|$ and $\psi$ using (2.1), (2.3), (2.4), $\Delta=\varepsilon^{-1}\left(\omega_{0}-2 \omega_{+}\right)$, (4.1) and (4.5). Thus, combining the two primary modes, we obtain

$$
\boldsymbol{u}=2 \varepsilon^{1 / 2}|a| \operatorname{Re}\left\{\boldsymbol{u}^{\left(\mu_{+}\right)}(\boldsymbol{X}) \exp \left[-\frac{1}{2} \mathrm{i}\left(\omega_{0} t+\psi+\frac{1}{2} \pi\right)\right]\right\}
$$

at leading order, where $\operatorname{Re}\{\}$ denotes the real part. Equation (4.6) shows that the spatial structure is determined by that of the primary mode, while there are fast-time oscillations at half the piston frequency and slow-time modulations of amplitude and phase owing to variation of $|a|$ and $\psi$. As discussed in Part 1, the primary mode, and hence the flow resulting from instability, consists of one or more oscillatory toroidal vortices encircling the cylinder axis. Although it is far from the only possible outcome, a fixed point of constant $|a|$ and $\psi$ may be approached at large times, yielding a flow which, according to (4.6), is phase locked to the piston oscillations with exactly half the piston frequency. The saturated amplitude and phase are determined by the $|a|$ and $\psi$ of the fixed point. Observe that the system (4.2), (4.3) is left invariant by a change of sign of $a$, corresponding to adding $2 \pi$ to $\psi$. Such a change in $\psi$ is equivalent to shifting time in (4.6) by the piston period, so the resulting flow (basic flow plus perturbation) is essentially the same for $-a(t)$ as for $a(t)$, only the time origin being different for the two flows. In particular, for each fixed point $a$, there is an equivalent one at $-a$.

Using (4.5) in (4.2), (4.3), we obtain the real system

$$
\begin{gathered}
\frac{\mathrm{d}|a|}{\mathrm{d} T}=-\left(\varepsilon^{-1} \overline{R e}^{-1 / 2} d_{+}^{r}+\omega_{+} C \cos \psi\right)|a|, \\
\frac{\mathrm{d} \psi}{\mathrm{d} T}=2 \omega_{+} C \sin \psi-\hat{\Delta}-2 G|a|^{2}-4 \sum_{\sigma \in M} \Lambda_{\mu_{+} \mu_{+} \sigma} \mathscr{A}_{\sigma}, \\
\frac{\mathrm{d} \mathscr{A}_{\sigma}}{\mathrm{d} T}=-\varepsilon^{-1} \overline{R e}^{-1 / 2} d_{\sigma} \mathscr{A}_{\sigma}+\left(2 C \Lambda_{\sigma \mu_{+} \mu_{+}} \cos \psi+\varepsilon^{-1} \overline{R e}^{-1 / 2} \Gamma_{\sigma}\right)|a|^{2},
\end{gathered}
$$

for $|a|, \psi$ and $\mathscr{A}_{\sigma}$. Equation (4.8) describes the time evolution of $\psi$, which governs the growth or decay of $|a|$ via (4.7). In (4.7), the first term in brackets represents primary mode damping, whereas the second corresponds to energy being fed into (or extracted from) the primary mode by piston motion. Both terms on the right-hand side of (4.7), as well as the first two on the right-hand side of (4.8), are already present in the linear theory, whereas $-2 G|a|^{2}$ and the geostrophic sum in (4.8) express the frequency shift of the primary mode discussed earlier. The two terms on the right-hand side of (4.9) correspond to viscous friction and nonlinear forcing acting on mode $\sigma$, effects also discussed earlier.

As noted in $\S 1$, if the viscous terms are dropped from (4.7)-(4.9), i.e. we set $\overline{R e}^{-1 / 2}=0$, the system is integrable. From (4.7) and (4.9), it is found that the quantities $\omega_{+} \mathscr{A}_{\sigma}+\Lambda_{\sigma \mu_{+} \mu_{+}}|a|^{2}$ are constant, allowing $\mathscr{A}_{\sigma}$ to be expressed in terms of $|a|^{2}$ and permitting elimination of the $\mathscr{A}_{\sigma}$ from (4.8). Dividing the result by (4.7) then yields a differential equation which can be integrated to express $\sin \psi$ as a function of $|a|^{2}$. The result is used in (4.7) to obtain a differential equation for $|a|^{2}$ as a function of $T$, whose solution may either be studied qualitatively in the phase-plane or expressed 
in terms of elliptic functions by quadrature. In general, the amplitudes $a$ and $\mathscr{A}_{\sigma}$ oscillate periodically with $T$, but, if the initial conditions are appropriately chosen, the solution is a homoclinic orbit associated with one of the unstable fixed points. An important special case is when the perturbation grows from the linear regime, corresponding to $a \rightarrow 0, \mathscr{A}_{\sigma} \rightarrow 0$ as $T \rightarrow-\infty$. Presuming linear instability of the basic flow represented by the fixed point $a=0, \mathscr{A}_{\sigma}=0$, the primary mode initially grows exponentially, while in the nonlinear regime $|a|$ and $\left|\mathscr{A}_{\sigma}\right|$ pass through a maximum and finally decay exponentially back to zero as $T \rightarrow+\infty$. Such behaviour, in which the basic flow is recovered in the end, is unexpected given the unstable nature of that flow. Small perturbations to the above solution introduce a certain amount of the exponentially growing component near the fixed point of zero $\boldsymbol{u}$ and it no longer goes back to zero. In particular, we would expect even small viscosity to modify the solution qualitatively, avoiding return to the basic flow. This behaviour is symptomatic of the singular nature of the inviscid problem, of which other aspects, such as the lack of a critical piston amplitude and the existence of unstable mode pairs of unboundedly small wavelengths, were discussed in Part 1 . It is apparent that viscosity must be included in both linear and nonlinear problems.

While we are on the subject of inviscid theories, as discussed in $\S 1$, Waleffe (1989) carried out an inviscid weakly nonlinear analysis of the different (but related, in that it involves instability due to parametric inertial-wave resonance) problem of an infinitely long rotating cylinder with small ellipticity (the elliptic instability). He obtained a pair of amplitude equations describing the primary mode and a mean-flow correction (equivalent to the combination $\mathscr{A}=\sum_{\sigma \in M} \Lambda_{\mu_{+} \mu_{+} \sigma} \mathscr{A}_{\sigma}$ discussed earlier) which have the same form as (4.2), (4.3) (after dropping the viscous terms and combining (4.3) as a single equation for $\mathscr{A}$ ). Like ours in the inviscid case, Waleffe's equations lead to periodic or fixed-point behaviour. He attempted to allow for viscosity by the expedient of adding linear damping terms to the inviscid amplitude equations, without carrying out the viscous analysis required in order to confirm such an approach. As is apparent from (4.2), (4.3), this is insufficient (at least in our case) because (a) there is nonlinear forcing catalysed by viscosity in (4.3), and $(b)$ (4.3) can no longer be combined as a single equation for $\mathscr{A}$ if volumetric damping of the geostrophic modes is allowed for.

It is evident from (4.7) that the primary mode amplitude decays monotonically to zero unless $\omega_{+}|C| \geqslant \varepsilon^{-1} \overline{R e}^{-1 / 2} d_{+}^{r}$. This condition corresponds to $\varepsilon \geqslant \varepsilon_{c}$, where $\varepsilon_{c}=d_{+}^{r} /\left(\overline{R e}^{1 / 2} \omega_{+}|C|\right)$ is the critical piston amplitude introduced in Part 1 , and below which linear instability does not occur no matter what the piston frequency. It now appears that, even when nonlinearity is allowed for, the primary mode amplitude decays for $\varepsilon<\varepsilon_{c}$, whereas when $\varepsilon>\varepsilon_{c}$, growth or decay of the primary mode depends on the phase $\psi$. Since, according to (4.9), the axisymmetric, geostrophic amplitudes passively follow the primary one, they also decay to zero as $T \rightarrow \infty$ when $\varepsilon<\varepsilon_{c}$, implying stability of the basic flow to perturbations of any magnitude for piston amplitudes less than $\varepsilon_{c}$. In the limiting case, $\varepsilon=\varepsilon_{c}$, it can be shown that, at large times, the solution approaches one of the zero or non-zero fixed points discussed later.

\subsection{Some numerical results}

In the absence of analytical solutions of (4.2), (4.3) (or equivalently (4.7)-(4.9)) we have used numerical integration. Given that the problem has four free parameters, namely $\overline{R e}, h_{0}, \hat{\Delta}$ (representing the piston frequency) and $\varepsilon$, together with the choice of primary mode and initial conditions, a systematic parametric study is hardly feasible and the cases examined were far from exhaustive. Examples are shown in figures 1 to 3. 
Figures 1 and 2 show trajectories of $a$ in the complex plane and corresponding plots of $|a|$ as a function of $t$ resulting from numerical integration with $\overline{R e}=10^{4}, h_{0}=2$ and $\hat{\Delta}=0$ for the lowest-order member of the family $n=0, m=1$ (a single toroidal vortex, the simplest case possible) and an initial perturbation small enough to be considered as growing from the linear regime. As will be seen, depending on the value of $\varepsilon$, the solution at large times approaches $(a)$ a fixed point, $(b)$ a limit cycle, or $(c)$ diverges to infinity. The latter outcome obviously invalidates the weakly nonlinear theory developed here, but at least indicates that the flow changes character significantly owing to the appearance of strong nonlinearity.

As the piston amplitude $\varepsilon$ is increased from zero, the first stability threshold crossed is the linear one, which lies at $\varepsilon=\varepsilon_{c}$ since $\hat{\Delta}=0$. As we saw above, according to the present theory, the basic flow is stable to perturbations of any amplitude below $\varepsilon=\varepsilon_{c}$. At $\varepsilon=\varepsilon_{c}(=0.076014$ in the present case $)$, the usual bifurcation associated with a stability boundary yields a locally stable non-zero fixed point (figures $1 a, 2 a$ ). In keeping with the discussion following (4.6), this fixed point represents a new flow consisting of an oscillatory toroidal vortex, phase-locked to the piston oscillations and with half the piston frequency. The fixed point later loses local stability, yielding a Hopf bifurcation to the limit cycle shown in figures $1(b)$ and $2(b)$, whose amplitude grows as $\varepsilon$ is further increased. Such a limit cycle corresponds to slow-time periodic modulation of the toroidal vortex, behaviour which persists until the limit cycle attains infinite amplitude and divergence occurs (figures $1 f$ and $2 f$ ) at and above a threshold piston amplitude ( $\varepsilon=0.10262$ in the present case).

Between the values of $\varepsilon$ at which the limit cycle first appears and then diverges, it undergoes a sequence of infinite-period bifurcations through $a=\mathscr{A}_{\sigma}=0$, the first of which is shown in figures $1(c)$ and 2(c). At each of these bifurcations, the limit cycle switches from being asymmetric under the invariance $a \mapsto-a$ of (4.2), (4.3) to being symmetric, or vice versa (see figures $1 b$ and $1 d$ for the first bifurcation and note that an asymmetric limit cycle is accompanied by a second, equivalent one obtained by switching the sign of $a$ ). The bifurcations also allow the winding number of the limit cycle about the origin to rise, leading to the spiral form illustrated by figure 1(e). Such a limit cycle consists of two phases, both apparent in figure 1(e) (and reflected in figure $2 e$ ). During the first phase, corresponding to the line sprouting out from the origin in figure 1(e), the perturbation grows as if divergence were going to occur, while in the second phase the perturbation spirals $(|a|$ decays, though not monotonically, as witnessed by the small wiggles in figure $2 e$ ) back to small amplitude and the cycle restarts owing to the instability of the basic flow. As noted earlier and is apparent from (4.7), growth or decay of the primary mode depends on the value of $\psi$, twice the angle between $a$ and the line $a_{r}=-a_{i}>0$ in figure $1 e$, which is roughly constant and appropriate for growth during the early stages of the limit cycle. The shift in the primary mode frequency owing to nonlinearity discussed earlier causes $\psi$ to evolve and subsequently to vary rapidly with time. This means that the term in (4.7) involving $\psi$ oscillates rapidly during the spiral phase and, in a time-averaged sense, contributes little to the evolution of $|a|$, which consequently decays owing to viscous damping. Given decreasing forcing by the primary mode, the geostrophic modes also decay. Once divergence occurs (figure $1 f$ ), although nonlinearity causes $\psi$ to evolve, rapid variation of $\psi$ does not take place and $\psi$ tends to a constant value compatible with continued exponential growth as $T \rightarrow \infty$.

Non-zero values of $\hat{\Delta}$ were found to exhibit similar behaviour as $\varepsilon$ increases, with an additional twist if $\hat{\Delta}<0$ : there can be intervals of $\varepsilon$ in which the oscillations become aperiodic, an example being given in figure 3 . It appears that aperiodicity arises in 

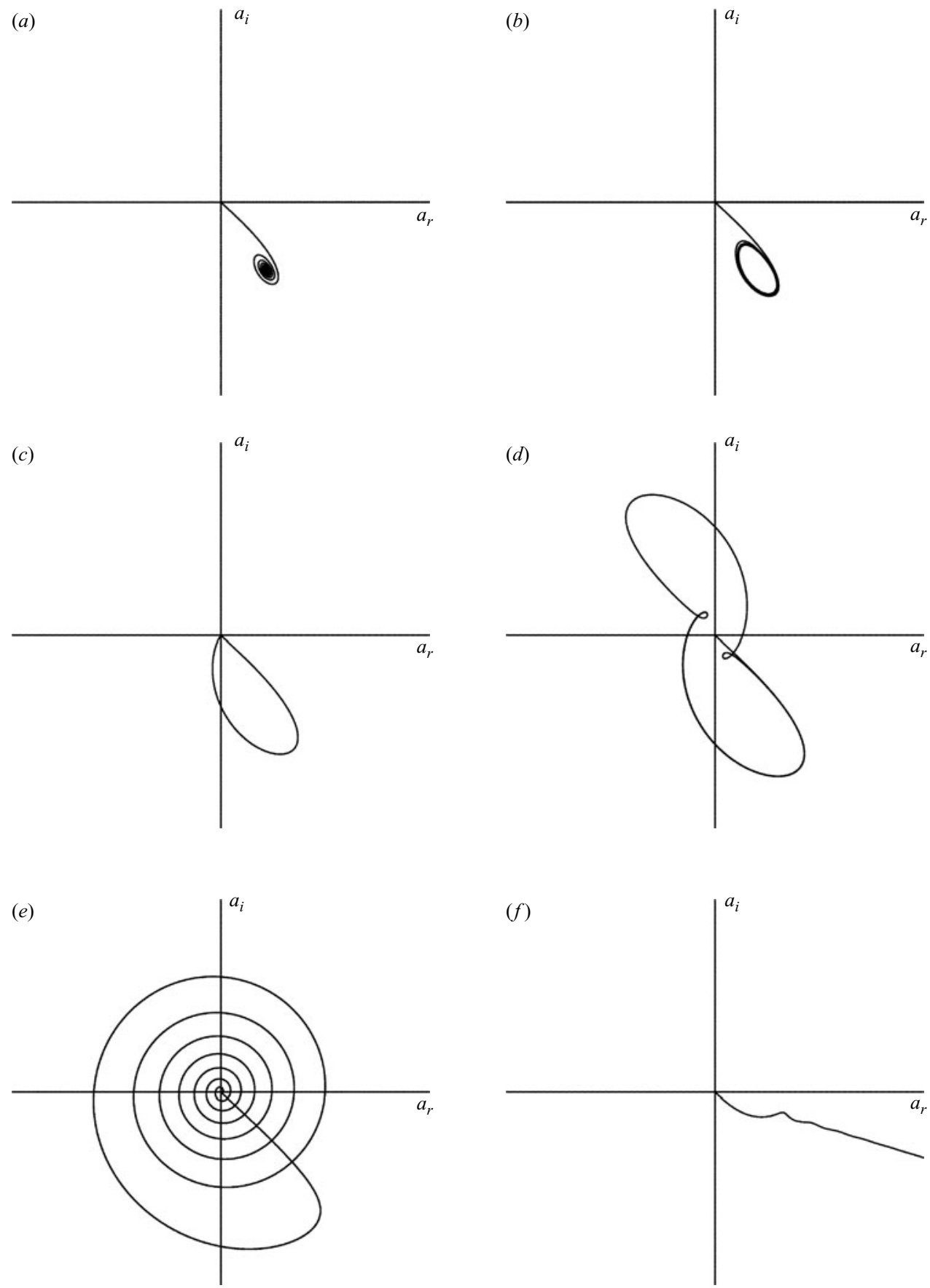

FIGURE 1. Trajectories in the complex $a$-plane obtained by numerical integration of (4.2), (4.3) with $\overline{R e}=10^{4}, h_{0}=2, \hat{\Delta}=0$ and the primary mode consisting of the lowest-order member of the family $n=0, m=1$. The values of $\varepsilon$ are $(a) \varepsilon=0.085$, (b) $\varepsilon=0.087$, (c) $\varepsilon=0.09051$, (d) $\varepsilon=0.093$, $(e) \varepsilon=0.102,(f) \varepsilon=0.17$. The initial modal amplitudes are $\mathscr{A}_{\sigma}=0$ and $|a|$ sufficiently small that the perturbation is initially in the linear regime. The part of the complex plane shown is $\left|a_{r}\right|<1,\left|a_{i}\right|<1$ for $(a-d)$ and $\left|a_{r}\right|<3,\left|a_{i}\right|<3$ for $(e, f)$. 
(a)

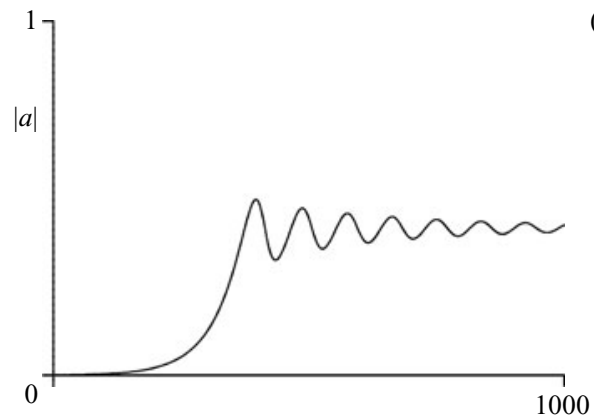

(c)

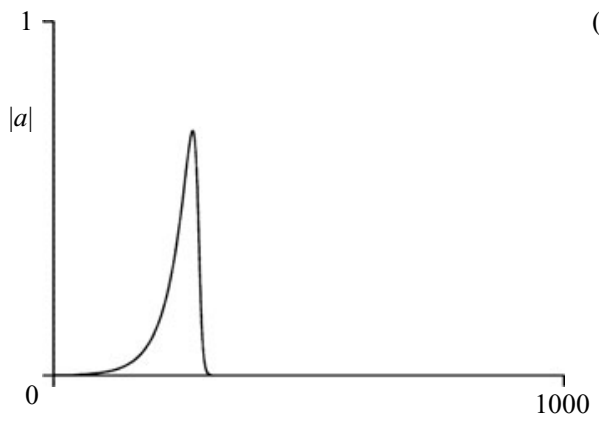

(b)

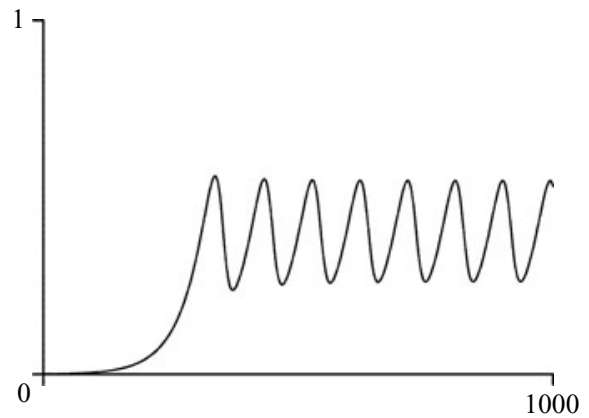

(1)

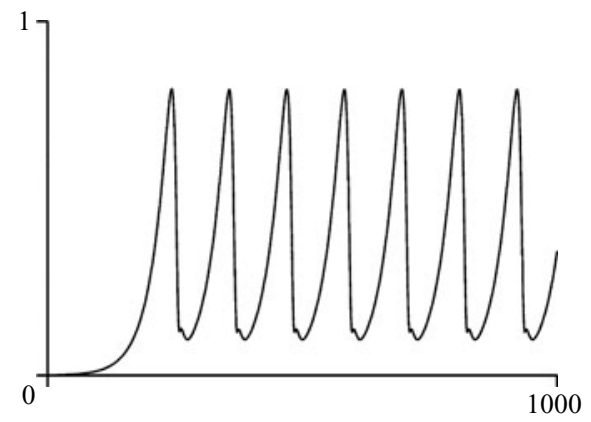

$(f)$

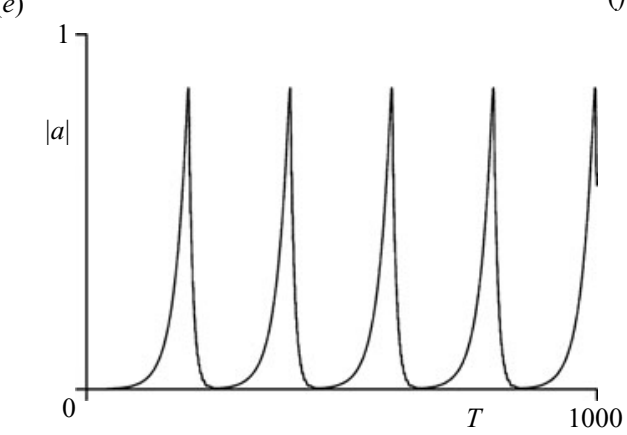

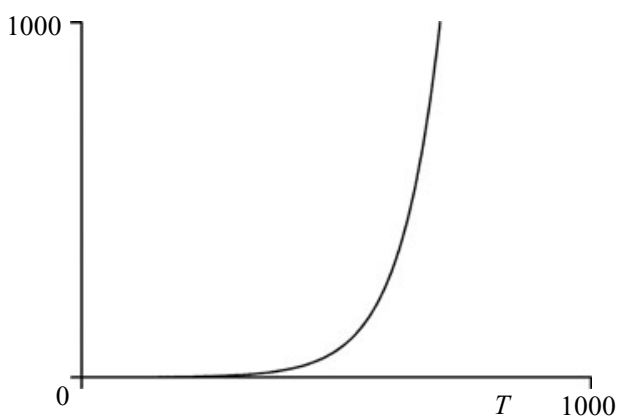

Figure 2. Time evolution of $|a|$ for the same cases as figure 1.

one of the usual ways via an infinite sequence of bifurcations. The usual signatures of mathematical chaos, namely a positive Lyapunov exponent and a broadband frequency spectrum, are found in the case of figure 3. The chaotic nature of the orbit manifests itself as apparently random variations in the length and maximum amplitude of successive cycles in figure $3(b)$, but the mechanisms underlying the oscillations are the same as described above for a limit cycle. As $\varepsilon$ is further increased for the case shown in figure 3 , divergence occurs, apparently without the need for the oscillations to approach infinite amplitude. Note that oscillatory regimes, both periodic and aperiodic, can occur above the threshold piston amplitude for divergence obtained in $\S 5.3(\varepsilon=0.10262$ for the case shown in figure 3$)$.

In order to have a less ad hoc description of at least some aspects of the dynamics than is possible from numerical examples such as the above, in the next two subsections we analyse the fixed points and divergences of the system (4.7)-(4.9). 

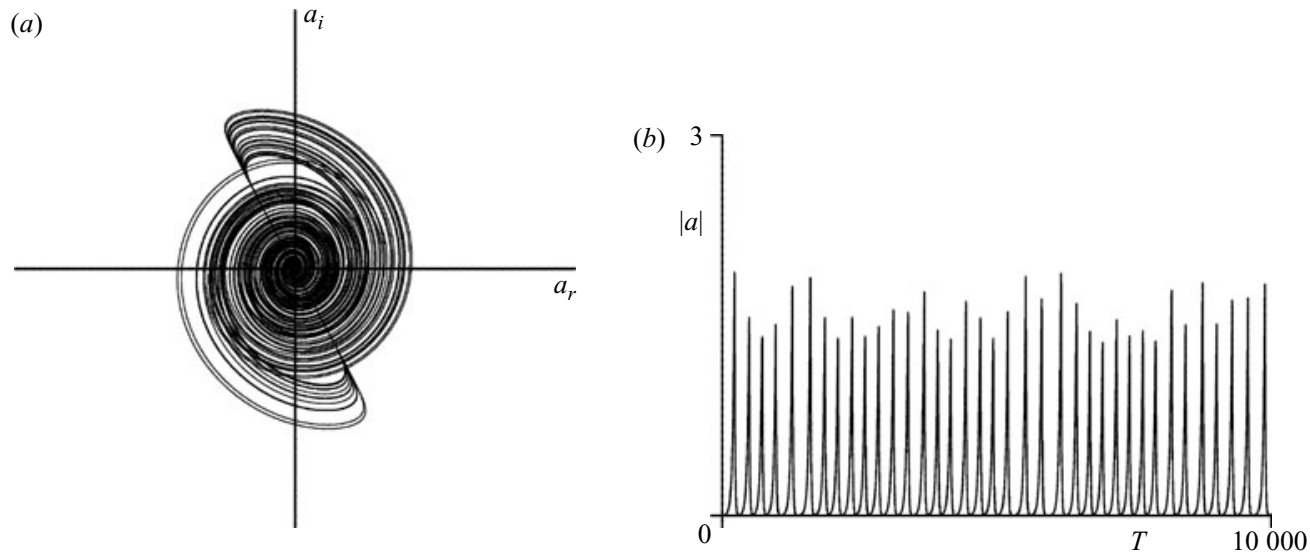

FiguRE 3. (a) The trajectory in the complex $a$-plane $\left(\left|a_{r}\right|<3,\left|a_{i}\right|<3\right)$, and $(b)$ the time evolution of $|a|$ with $\overline{R e}=10^{4}, h_{0}=2, \hat{\Delta}=-0.2, \varepsilon=0.130255$ and the primary mode consisting of the lowest-order member of the family $n=0, m=1$. The initial modal amplitudes are $\mathscr{A}_{\sigma}=0$ and $|a|$ sufficiently small that the perturbation is initially in the linear regime.

\subsection{Fixed points}

\section{Fixed-point branches}

The basic flow, $a=\mathscr{A}_{\sigma}=0$, always yields a fixed point. As discussed earlier, for each non-zero fixed point there is an equivalent one obtained by changing the sign of $a$. Restricting attention to non-zero fixed points in $a_{r} \geqslant 0$ and setting the right-hand side of (4.7) to zero, we find that $\psi= \pm \psi_{0}$ where $\psi_{0}$ is the solution of

$$
\cos \psi_{0}=-\frac{\varepsilon^{-1} \overline{R e}^{-1 / 2} d_{+}^{r}}{\omega_{+} C}=\frac{\varepsilon_{c}}{\varepsilon}
$$

in $0 \leqslant \psi_{0}<\pi / 2$. Thus, a prerequisite for a non-zero fixed point is $\varepsilon \geqslant \varepsilon_{c}$. Setting the right-hand side of (4.9) to zero and using (4.10) yields

$$
\mathscr{A}_{\sigma}=\frac{\Gamma_{\sigma}-2 \omega_{+}^{-1} d_{+}^{r} \Lambda_{\sigma \mu_{+} \mu_{+}}}{d_{\sigma}}|a|^{2},
$$

which determines $\mathscr{A}_{\sigma}$ in terms of $|a|$. Setting the right-hand side of (4.8) to zero and using (4.10) and (4.11), we obtain

$$
|a|=\left(-\frac{\hat{\Delta} \pm \Delta_{0}}{2 \tilde{G}}\right)^{1 / 2}
$$

in which the choice of signs corresponds with that of $\psi= \pm \psi_{0}, \Delta_{0}=$ $-2 \omega_{+} C\left(1-\left(\varepsilon_{c} / \varepsilon\right)^{2}\right)^{1 / 2}$ yields the linearly unstable band of piston frequencies as $|\hat{\Delta}|<\Delta_{0}$, and

$$
\tilde{G}=G+2 \sum_{\sigma \in M} \frac{\Lambda_{\mu_{+} \mu_{+} \sigma}}{d_{\sigma}}\left(\Gamma_{\sigma}-\frac{2 d_{+}^{r}}{\omega_{+}} \Lambda_{\sigma \mu_{+} \mu_{+}}\right)
$$

is a coefficient which depends only on $\overline{R e}, h_{0}$ and the choice of primary mode. In the singular case $\tilde{G}=0$, non-zero fixed points do not exist. Otherwise, they exist provided the argument of the square root in (4.12) is positive, with amplitude given 

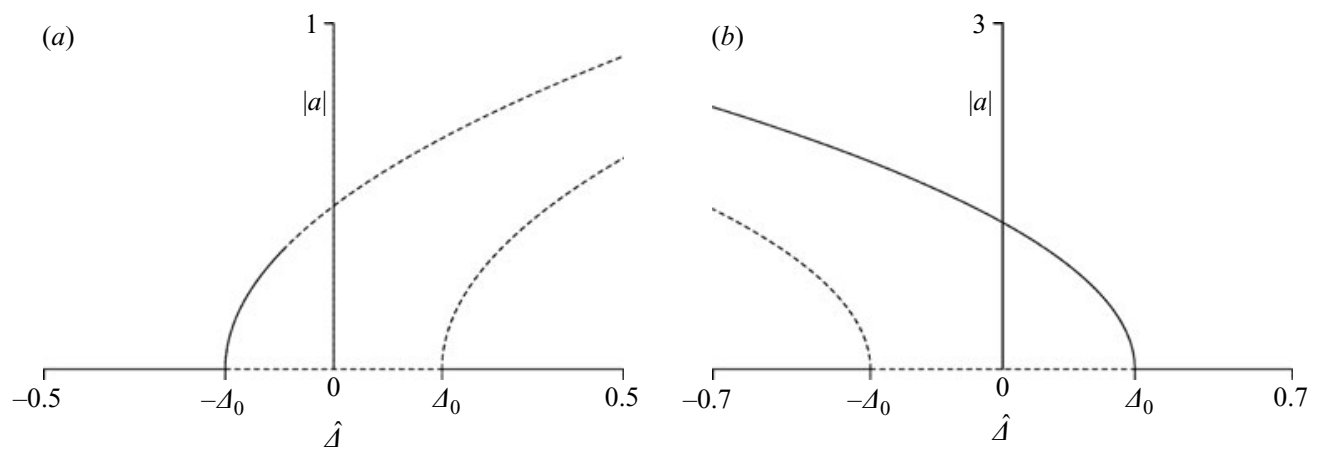

FIGURE 4. Plots of the fixed-point amplitudes as a function of $\hat{\Delta}$ for the primary mode consisting of the lowest-order member of the family $n=0, m=1$ with $(a) \overline{R e}=10^{4}, h_{0}=2$, $\varepsilon=0.093$, and $(b) \overline{R e}=2 \times 10^{4}, h_{0}=0.8, \varepsilon=0.2$. The continuous lines show locally stable fixed points, the dashed ones indicate unstable fixed points.

by (4.12) and phase $\psi= \pm \psi_{0}$. Note that non-zero fixed points always lie in the quadrant $a_{r}>0, a_{i}<0$ of the complex $a$-plane (with an equivalent one in $a_{r}<0$, $a_{i}>0$ obtained via the symmetry $\left.a \mapsto-a\right)$ and have phase determined by setting the right-hand side of (4.7) to zero. Since the terms on the right-hand side of (4.7) originate from linear terms in (4.2) (namely the piston-motion and viscous damping terms), the phase is controlled by linear mechanisms, even though the existence of non-zero fixed points requires nonlinearity.

Figure 4(a) shows graphs of the fixed-point amplitudes as functions of $\hat{\Delta}$ for a particular case (corresponding to figure $1 d$, but with $\hat{\Delta}$ now allowed to vary) in which $\tilde{G}$ is negative. The two parabolas shown in the figure represent non-zero fixed points which are born by bifurcation when the basic flow loses linear stability at the edges of the unstable band, $|\hat{\Delta}|<\Delta_{0}$, of piston frequencies. If $\tilde{G}$ is positive, as in figure $4(b)$, the parabolas branch off from the band edges in the opposite sense. Continuous lines in these bifurcation diagrams represent locally stable fixed points, whereas dashed lines correspond to unstable ones. Notice that the two non-zero fixed points merge when $\varepsilon=\varepsilon_{c}$ (leading to $\Delta_{0}=0$ and coincidence of the two parabolas in the bifurcation diagram), before disappearing when $\varepsilon<\varepsilon_{c}$. As noted earlier, when $\varepsilon=\varepsilon_{c}$, the solution can be shown to approach one of the fixed points (possibly the basic flow) as $T \rightarrow \infty$. For this reason, we restrict attention to $\varepsilon>\varepsilon_{c}$ in what follows.

\section{Local stability of fixed points}

Analysis of local stability, based on linearization of (4.7)-(4.9) about a non-zero fixed point, is straightforward and only briefly sketched here. We look for solutions of the linearized equations of the exponential form $\exp \left(s T / \varepsilon \overline{\operatorname{Re}}^{1 / 2}\right)$ and obtain a matrix eigenvalue problem for $s$ which was solved using a standard eigenvalue routine when plotting figure 4. As usual, an eigenvalue with a positive real part is symptomatic of instability and leads to a dashed line in the bifurcation diagram. For analytical purposes, the eigenvalues may be shown to be the complex roots of $f(s)=0$, where

$$
f(s)=s\left(s+2 d_{+}^{r}\right)+\alpha\left(G+2 \sum_{\sigma \in M} \frac{\Lambda_{\mu_{+} \mu_{+} \sigma}}{s+d_{\sigma}}\left(\Gamma_{\sigma}-\frac{s+2 d_{+}^{r}}{\omega_{+}} \Lambda_{\sigma \mu_{+} \mu_{+}}\right)\right)
$$

and $\alpha=\mp 2 \varepsilon^{2} \overline{R e} \Delta_{0}|a|^{2}$ is the only parameter via which dependence on $\varepsilon$ and $\hat{\Delta}$ enters the problem. The function $f(s)$ is real for real $s$, going from $f(0)=\alpha \tilde{G}$ 
to $f(+\infty)=+\infty$ as $s$ runs over positive real values. For the lower branch in the bifurcation diagram, $\alpha \tilde{G}<0$, so that the branch yields a real positive zero of $f(s)$ and is consequently always unstable, as reflected in figure 4 . As is also seen from figure 4 , the upper branch may or may not lose stability as $\hat{\Delta}$ is varied. At a point of stability loss, $f(s)$ has a zero on the imaginary $s$-axis. Since $f(0)=\alpha \tilde{G}$, loss of stability is always oscillatory because otherwise $\tilde{G}=0$, the singular case noted earlier for which the fixed point does not exist.

The upper branch is locally stable near its bifurcation point at $\hat{\Delta}=\Delta_{0} \operatorname{sgn}(\tilde{G})$, as is apparent by examining the $\alpha \rightarrow 0$ limit of the complex zeros of (4.14), which behave as $s \rightarrow-2 d_{+}^{r}, s \rightarrow-d_{\sigma}$ and $s \sim-\alpha \tilde{G} / 2 d_{+}^{r}$ and are all of negative real part since $\alpha \tilde{G}>0$ for the upper branch. Varying either $\hat{\Delta}$ or $\varepsilon$ changes (4.14) via $\alpha$. Thus, for given $\overline{R e}, h_{0}$ and a particular choice of primary mode, stability depends on just the one parameter $\alpha$, which has the same sign as $\tilde{G}$, but can otherwise take on any value. Varying $\alpha$ and solving the matrix eigenvalue problem, the upper branch fixed point is either stable for all $\alpha$, and hence for all $\hat{\Delta}$ and $\varepsilon$ (as in figure $4 b$ ), or it has one or more stability thresholds. In a systematic (but non-exhaustive) parametric search, in which $\overline{R e}, h_{0}$ and the primary mode were varied, we never found more than one threshold, so, for given $\overline{R e}, h_{0}$ and a particular choice of primary mode, the upper branch is either always locally stable or there is a single stability boundary, $\alpha=\alpha_{c}$. If $\alpha_{c}$ is such a threshold, then

$$
\hat{\Delta}=\operatorname{sgn}(\tilde{G})\left(\Delta_{0}-\frac{\alpha_{c} \tilde{G}}{\varepsilon^{2} \overline{R e} \Delta_{0}}\right)
$$

gives the corresponding stability boundary for $\hat{\Delta}$. Recalling that $\Delta_{0}=$ $-2 \omega_{+} C\left(1-\left(\varepsilon_{c} / \varepsilon\right)^{2}\right)^{1 / 2},(4.15)$ yields a neutral curve of the upper-branch fixed point in the $(\hat{\Delta}, \varepsilon)$-plane. Figure 5 shows a plot of this curve for the case of figure $4(a)$ (but now allowing $\varepsilon$ to vary). Because $\tilde{G}<0$ and $\Delta_{0}$ is a positive, increasing the function of $\varepsilon$, from zero at $\varepsilon=\varepsilon_{c}$ to $-2 \omega_{+} C$ as $\varepsilon \rightarrow \infty$, (4.15) decreases from $+\infty$ at $\varepsilon=\varepsilon_{c}$ to $2 \omega_{+} C$ as $\varepsilon \rightarrow \infty$. As $\varepsilon$ increases, the neutral curve of the upper branch approaches the left-hand side of the basic-flow neutral curve, $|\hat{\Delta}|=\Delta_{0}$, which is also shown in figure 5 , as is the divergence threshold, $\varepsilon=\varepsilon_{d}$, derived in the next subsection. It should be borne in mind that the upper-branch fixed point, whose local stability we are considering, exists only for $\varepsilon \geqslant \varepsilon_{c}$ and $\hat{\Delta}>-\Delta_{0}\left(\hat{\Delta}<\Delta_{0}\right.$ if $\left.\tilde{G}>0\right)$, i.e. inside and to the right (left if $\tilde{G}>0$ ) of the neutral curve of the basic flow.

\section{Bifurcation type}

The nature, subcritical or supercritical, of the bifurcation associated with loss of stability of the upper branch may be determined in the usual way via weakly nonlinear analysis of (4.7)-(4.9) near the fixed point, the sign of the real part of the Landau constant determining the bifurcation type. Given the lengthy analysis, we do not give details here. It is found that the Landau constant consists of a positive factor multiplying a quadratic in $\varepsilon^{2}$ whose coefficients depend on $\overline{R e}, h_{0}$ and the choice of primary mode. Thus, for given $\overline{R e}, h_{0}$ and a particular primary mode, the coefficients of the quadratic can be calculated and hence the behaviour of the bifurcation determined as a function of $\varepsilon$. The systematic parametric search referred to above, in which $\overline{R e}, h_{0}$ and the primary mode were varied, led to the conclusion that (aside from some cases with values of $\varepsilon$ which are so large as to lie well beyond the scope of the present theory) the bifurcation is always supercritical. Given its oscillatory nature, the bifurcation yields a limit cycle, as illustrated by figures 1(a) 


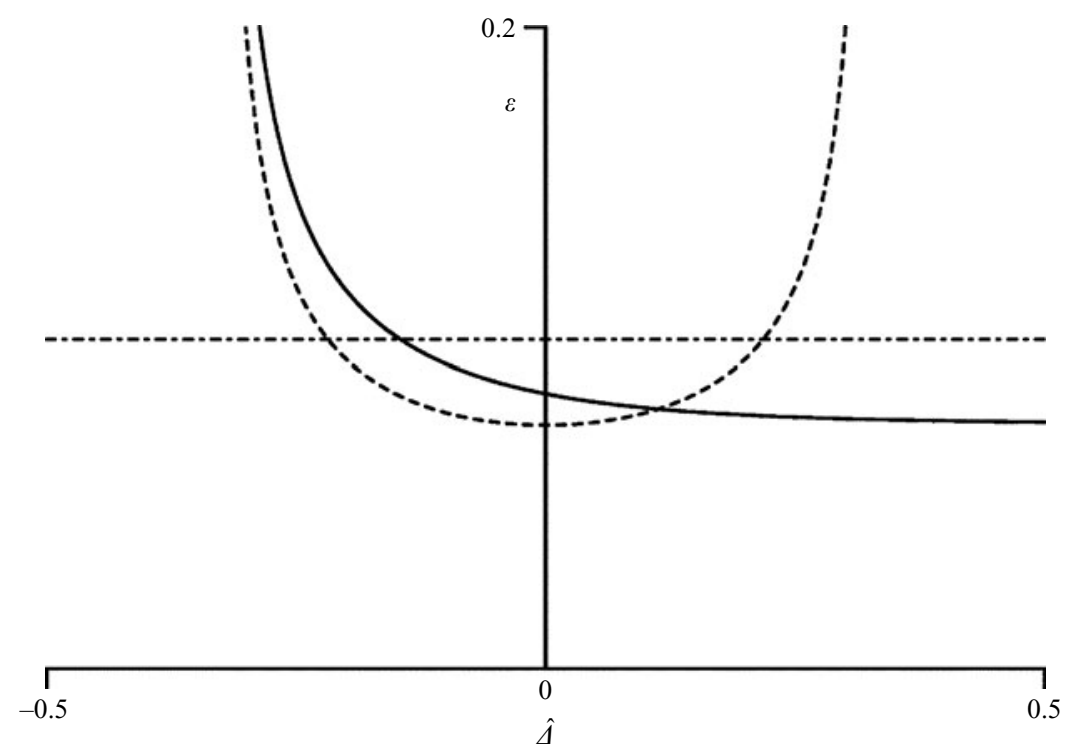

FIGURE 5. Neutral curves in the $(\hat{\Delta}, \varepsilon)$ plane for the upper-branch fixed point (continuous curve) and basic flow (dashed curve) with $\overline{R e}=10^{4}, h_{0}=2$ and the primary mode consisting of the lowest-order member of the family $n=0, m=1$. The dashed horizontal line is the divergence threshold $\varepsilon_{d}$.

and $1(b)$, which, as we saw earlier, may subsequently become aperiodic. It is therefore reasonable to conjecture, as found in our numerical simulations, that instability of the upper branch is a necessary condition for the existence of oscillations as $T \rightarrow \infty$. That is, such oscillations should arise only above the upper-branch neutral curve, otherwise a fixed point or divergence is to be expected.

\subsection{Divergence threshold}

In all cases in which the numerical solution diverged to infinity, it was observed to be accompanied by $\psi \rightarrow \psi_{\infty}$ (see e.g. figure $1 f$ ). Assuming this is the case in general, we derive a necessary condition for divergence. Equation (4.7) then implies that

$$
|a|^{2} \sim K \exp \left(s T / \varepsilon \overline{R e}^{1 / 2}\right)
$$

as $T \rightarrow \infty$, where

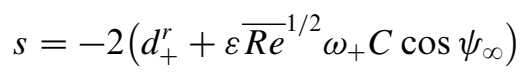

must be positive in order that $|a|$ grows, rather than decays. Using (4.16), (4.17) and $\psi \rightarrow \psi_{\infty}$, (4.9) leads to

$$
\mathscr{A}_{\sigma} \sim K \exp \left(s T / \varepsilon \overline{\operatorname{Re}}^{1 / 2}\right) \frac{\Gamma_{\sigma}-\omega_{+}^{-1}\left(s+2 d_{+}^{r}\right) \Lambda_{\sigma \mu_{+} \mu_{+}}}{s+d_{\sigma}} .
$$

Note that (4.16) and (4.18) show that divergence, when it occurs, is exponential. Using (4.16) and (4.18) in the right-hand side of (4.8), the coefficient of $\exp \left(s T / \varepsilon \overline{R e}^{1 / 2}\right)$ must 


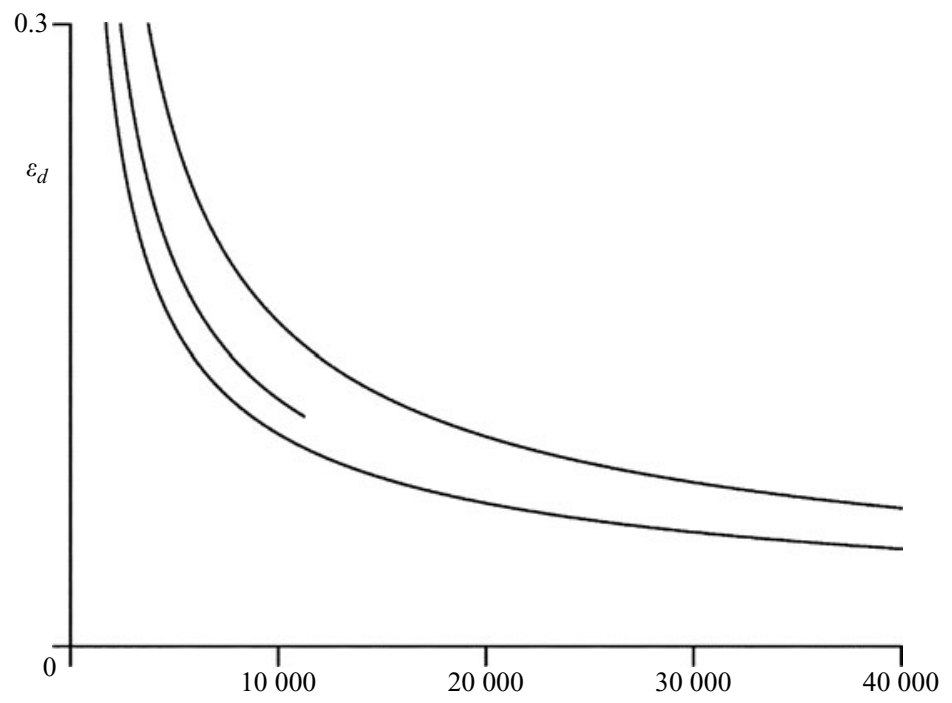

FiguRE 6. Plots of the divergence threshold $\varepsilon_{d}$ as a function of $\overline{R e}$ for the primary mode consisting of the lowest-order member of the family $n=0, m=1$ and three values of $h_{0}$ : the upper curve is for $h_{0}=10$, the lower curve for $h_{0}=2$, and the middle curve for $h_{0}=0.8$.

be zero, for otherwise $\psi$ would not approach a limit. Thus, we obtain $g(s)=0$, where

$$
g(s)=G+2 \sum_{\sigma \in M} \frac{\Lambda_{\mu_{+} \mu_{+} \sigma}\left(\Gamma_{\sigma}-\omega_{+}^{-1}\left(s+2 d_{+}^{r}\right) \Lambda_{\sigma \mu_{+} \mu_{+}}\right)}{s+d_{\sigma}}
$$

depends only on $s, \overline{R e}, h_{0}$ and the choice of primary mode.

In order that a zero of $g(s)$ can produce divergence, it must be positive. Thus, a prerequisite for divergence is that there be at least one such zero of $g(s)$. Furthermore, (4.17) should have a solution for $\psi_{\infty}$, i.e. the zero must lie in

$$
s \leqslant 2\left(\varepsilon \overline{\operatorname{Re}}^{1 / 2} \omega_{+}|C|-d_{+}^{r}\right),
$$

a condition which is satisfied provided the right-hand side exceeds the smallest positive zero, $s_{\min }$, of $g(s)$. Thus, divergence requires that $\varepsilon \geqslant \varepsilon_{d}$, where

$$
\varepsilon_{d}=\frac{s_{\min }+2 d_{+}^{r}}{2 \overline{R e}^{1 / 2} \omega_{+}|C|}
$$

is a threshold piston amplitude for divergence (which can be extended to the case when $g(s)$ does not have positive zeros by setting $\varepsilon_{d}=+\infty$ in that case). Since the function $g$ depends only on $\overline{R e}, h_{0}$ and the particular choice of primary mode, $\varepsilon_{d}$ is independent of $\hat{\Delta}$, as reflected by the horizontal dashed line in figure 5 representing the divergence threshold $\varepsilon=\varepsilon_{d}$. Observe that, since $\varepsilon_{c}=d_{+}^{r} / \overline{\operatorname{Re}}^{1 / 2} \omega_{+}|C|,(4.21)$ implies that $\varepsilon_{d}>\varepsilon_{c}$, which is an obvious consequence of (4.7) not allowing growth of $|a|$ when $\varepsilon \leqslant \varepsilon_{c}$.

Figure 6 shows $\varepsilon_{d}$ as a function of $\overline{R e}$ for three values of $h_{0}$ and the same primary mode as for earlier figures. It is apparent that $\varepsilon_{d}$ decreases with increasing $\overline{R e}$, implying that larger Reynolds numbers tend to favour divergence. On the other hand, the curve for $h_{0}=0.8$ comes to an abrupt end at a certain value of $\overline{R e}$, above 
which $g(s)$ has no positive zeros and divergence cannot occur. Thus, in this rather different sense, larger Reynolds numbers may inhibit divergence.

The condition, $\varepsilon \geqslant \varepsilon_{d}$, for divergence is necessary but not sufficient. For instance, the basic flow is locally stable outside its neutral curve in figure 5. Thus, a perturbation which approaches zero, rather than diverging, is possible even when $\varepsilon \geqslant \varepsilon_{d}$. On the other hand, divergence occurs in figures 1 and 2 as soon as $\varepsilon \geqslant \varepsilon_{d}$. More generally, provided $\varepsilon \geqslant \varepsilon_{d}$, we found that the initial conditions could always be chosen to make divergence happen. Thus, as is often the case, a number of outcomes are possible and which one actually occurs depends on the initial conditions via the attraction basins of the various possibilities. In our case, these possibilities appear to consist of the fixed points identified earlier (when they are locally stable), a limit cycle or chaotic attractor when the upper-branch fixed point is unstable, and divergence if $\varepsilon \geqslant \varepsilon_{d}$.

\section{Conclusions}

Weakly nonlinear amplitude equations for the primary modes resulting from linear instability have been derived. They contain an infinity of axisymmetric geostrophic mode amplitudes, representing a modification to the mean flow which, along with cubic interactions among the primary modes, produces a small, but significant, shift in their frequencies. This frequency shift causes the evolution of the primary mode phase, which controls the rate and direction of energy supply to the primary modes by piston motion, to evolve differently and hence affects modal growth or decay. The geostrophic amplitude equations show mean forcing coming from quadratic interactions of the primary modes, catalysed by piston motion and viscosity, and resisted by viscous damping. Thus, as the primary modes grow from the linear regime, the geostrophic modes are spun up, leading to nonlinear effects on the primary modes owing to frequency shifting once the perturbation has reached $O\left(\varepsilon^{1 / 2}\right)$, large compared with the basic flow due to piston oscillations of $O(\varepsilon)$. We expect weakly nonlinear amplitude equations of the same generic form (but representing different primary modes and with different coefficients) for other instabilities arising from parametric resonance of inertial modes.

The detailed consequences of the amplitude equations have been investigated in the case of an axisymmetric primary mode, corresponding to one or more oscillatory toroidal vortices encircling the cylinder axis, and axisymmetric geostrophic flow. The inclusion of viscosity was found to be crucial to avoid singular behaviour arising from integrability of the inviscid equations. The geostrophic modes were also found to be an essential ingredient of the problem, for without them the solution always approaches a fixed point at large times, representing, in the case of a non-zero fixed point, a saturated state in which the primary mode becomes phase-locked to the piston motion with half its frequency. The full set of equations also allow such fixed points as $T \rightarrow \infty$ for some ranges of parameter values and initial conditions, but much richer behaviour is possible: periodic or chaotic oscillations, or exponential divergence of the mode amplitudes to infinity. The latter outcome invalidates the weakly nonlinear theory used here, but shows that the character of flow must change dramatically, perhaps undergoing finite-amplitude wave breaking and transition to turbulence, as observed in a number of experiments. In any case, divergence provides a route out of the weakly nonlinear regime, while a limit cycle or aperiodic attractor implies slow-time oscillations of the amplitude and phase of the primary and geostrophic modes. 
In addition to the zero fixed point of the amplitude equations representing the basic flow, which was shown to be globally stable for piston amplitudes below the critical value $\varepsilon_{c}$ from linear theory and is locally unstable inside the neutral curve in the piston frequency-amplitude plane determined in Part 1, two non-zero fixed-point branches (referred to as lower and upper branches according to the magnitude of the associated perturbation amplitude) are born via pitchfork bifurcation when the basic flow loses local stability as the neutral curve is crossed. Local stability analysis of these non-zero fixed points showed that the lower branch is always unstable, whereas the upper branch, although stable near its bifurcation from the basic flow, may subsequently lose stability, leading to a supercritical Hopf bifurcation and a limit cycle. Chaotic oscillations were subsequently observed to appear via a sequence of bifurcations, and it was conjectured that such oscillations, whether periodic or not, exist only when the upper branch is unstable. During oscillations of large maximum amplitude, the perturbation initially grows from small values owing to linear instability of the basic flow, but nonlinearity intervenes to detune the parametric resonance, leading to decay back from large amplitudes during the second half of the cycle. The perturbation returns to small amplitude and the process begins again.

Analysis of divergence led to a threshold piston amplitude, dependent on the rotational Reynolds number, cylinder geometry and choice of primary mode, but independent of the piston frequency, below which divergence cannot occur. The threshold may be infinite, meaning that divergence cannot occur no matter what the piston amplitude and frequency. Once the threshold is exceeded, it always seems possible to choose the initial conditions so that divergence happens, but cases were found in which a fixed point, limit cycle or aperiodic attractor was approached at large times, despite being above the divergence threshold.

Following on from the analytical work described here and in Part 1, experimental (Graftieaux et al. 2002; Graftieaux 2003) and DNS (Duguet, Scott \& Le Penven 2005) studies of the present instability for the lowest-order axisymmetric mode have confirmed the neutral curve obtained in Part 1 and the end-result of instability as the predicted inertial mode. They also found that the frequency spectrum of the velocity field, consisting of the piston frequency and its harmonics in the absence of instability, acquired a strong peak at $\omega_{0} / 2$, more energetic than that at $\omega_{0}$, when instability was present. This is consistent with the asymptotic dominance of the perturbation $\left(O\left(\varepsilon^{1 / 2}\right)\right)$ over the basic flow $(O(\varepsilon))$ in the present theory. Both studies found that, depending on parameter values, the amplitude of the primary mode either approached a limiting value or underwent slow (compared to the piston period) oscillations at long times, outcomes corresponding to the fixed points and slow-time limit cycles of the present work, though the amplitude of modal amplitude oscillations in Duguet et al. (2005) was smaller than predicted by the present theory. Duguet et al. (2005) (and Le Penven in an as yet unpublished analysis of Graftieaux's data) also determined the mean flow in the case of a fixed point and found reasonable agreement with the results of the present theory. Since the mean flow contains the geostrophic modes, this provides a detailed check on the theory. Note however, that no clear equivalent of divergence was found (nor any flow breakdown to turbulence). In summary, although the linear theory of Part 1 has been confirmed, questions remain on the nonlinear side. We aim to resolve these issues in future experimental work.

As noted in $\S 1$, the experimental study by Eloy et al. (2003) of the elliptic instability found either a fixed point or flow breakdown (they also found cases in which a secondary parametric instability occurred; see the discussion below). For the case of a fixed point, they found good agreement between the experimental and weakly 
nonlinear theoretical amplitude and phase of the primary mode, the latter being obtained using amplitude equations for the elliptic case of the same form as derived by asymptotic analysis here.

Finally, in deriving the geostrophic amplitude equation (3.12), it was supposed, for simplicity, that a secondary, symmetry-breaking, geostrophic instability does not occur, otherwise the geostrophic flow would spontaneously become non-axisymmetric and the more general equation, (B 12), would be required. Kerswell (1999) identified such a geostrophic instability by linear stability analysis of a single inertial mode in a cylinder (with the simplifying assumptions of constant modal amplitude, neglect of the axisymmetric geostrophic flow it would induce, and artificial stress-free boundary conditions on the endwalls (as a result, Kerswell's solubility condition for his equation (4.7) is equivalent to the eigenvalue problem for the matrix $\left.\mathrm{i} \Xi_{\sigma \lambda}\right)$ ). Kerswell (1999) and Mason \& Kerswell (1999) also found another type of secondary instability, implicitly supposed absent in our analysis, in which two secondary modes are such that their frequency difference is close to the frequency of the primary mode (cf. the discussion in Kerswell 2002, §4). This is a parametric instability, similar in nature to the primary one, but in which the primary mode plays the role of the piston oscillations in our problem or ellipticity in the elliptic one. Eloy et al. (2003) observed this kind of instability experimentally in an elliptic cylinder for certain parameter values. Clearly, secondary instabilities can be important and merit further study, but are beyond the scope of the present work.

J.-P. R. would like to acknowledge support by the Service de la Recherche et des Etudes Amont for a part of the study reported in this paper.

\section{Appendix A. Nonlinear coefficients for axisymmetric modes}

This Appendix gives detailed expressions for the coefficients $G, \Lambda_{\sigma \mu_{+} \mu_{+}}, \Lambda_{\mu_{+} \mu_{+} \sigma}$ and $\Gamma_{\sigma}$ appearing in the amplitude equations. Throughout, $\mu_{+}$is axisymmetric and $\sigma$ both axisymmetric and geostrophic. Derivation of (A 1)-(A 3) can be found in Appendix $\mathrm{E}$, while (A 4) is derived in Appendices $\mathrm{F}$ to $\mathrm{H}$. The integrals appearing below are evaluated numerically.

$$
\begin{gathered}
G=\frac{\omega_{+}}{3 \pi h_{0} J_{0}^{4}\left(k^{\left(\mu_{+}\right)}\right)} \int_{0}^{k^{\left(\mu_{+}\right)}}\left(\frac{10}{\xi}-\xi\right) J_{0}^{\prime 4}(\xi) \mathrm{d} \xi \\
\Lambda_{\sigma \mu_{+} \mu_{+}}=\frac{2 \omega_{+} k^{\left(\mu_{+}\right)}}{\left(\pi h_{0}\right)^{1 / 2} J_{0}^{2}\left(k^{\left(\mu_{+}\right)}\right) J_{0}\left(k^{(\sigma)}\right)} \int_{0}^{1} r J_{0}\left(k^{\left(\mu_{+}\right)} r\right) J_{0}^{\prime}\left(k^{\left(\mu_{+}\right)} r\right) J_{0}^{\prime}\left(k^{(\sigma)} r\right) \mathrm{d} r, \\
\Lambda_{\mu_{+} \mu_{+} \sigma}=\frac{3 k^{\left(\mu_{+}\right) 2}-k^{(\sigma) 2}}{2 k^{\left(\mu_{+}\right) 2}} \Lambda_{\sigma \mu_{+} \mu_{+}}, \\
\Gamma_{\sigma}=\left(\beta^{(1)}+\beta^{(2)} k^{(\sigma) 2}\right) \Lambda_{\sigma \mu_{+} \mu_{+}}, \\
\beta^{(1)}=\frac{4}{h_{0} \omega_{+}}\left(2^{1 / 2}\left[\frac{1}{4}\left(1-\omega_{+}^{2}\right)\left(h_{0} \omega_{+}^{1 / 2}-\frac{\left(1-\omega_{+}^{2}\right)^{1 / 2}}{\omega_{+}}\left(\left(1+\omega_{+}\right)^{1 / 2}-\left(1-\omega_{+}\right)^{1 / 2}\right)\right)-1\right]\right. \\
+\operatorname{Re}\left\{\int _ { 0 } ^ { \infty } \operatorname { e x p } ( - 2 ^ { - 1 / 2 } \xi ) \left[\left(\mathscr{F}^{*} \frac{\mathrm{d} F_{\theta}}{\mathrm{d} \xi}-F_{r}^{*} F_{\theta}\right) \cos \left(2^{-1 / 2} \xi\right)\right.\right. \\
\left.\left.\left.-\left(\mathscr{F}^{*} \frac{\mathrm{d} F_{r}}{\mathrm{~d} \xi}+\left|F_{r}\right|^{2}+2\left|F_{\theta}\right|^{2}-\omega_{+}^{2}-2\right) \sin \left(2^{-1 / 2} \xi\right)\right] \mathrm{d} \xi\right\}\right) .
\end{gathered}
$$




$$
\begin{gathered}
\beta^{(2)}=\frac{2}{h_{0} \omega_{+} k^{\left(\mu_{+}\right) 2}}\left(2^{-1 / 2}+\int_{0}^{\infty} \exp \left(-2^{-1 / 2} \xi\right)\left(\left|F_{r}\right|^{2}+\left|F_{\theta}\right|^{2}-\omega_{+}^{2}-1\right) \sin \left(2^{-1 / 2} \xi\right) \mathrm{d} \xi\right) \\
F_{r}(\xi)=\mathrm{i}\left(\omega_{+}-\frac{1}{2}\left(\left(1+\omega_{+}\right) \exp \left(-\gamma_{-}^{\left(\mu_{+}\right)} \xi\right)-\left(1-\omega_{+}\right) \exp \left(-\gamma_{+}^{\left(\mu_{+}\right)} \xi\right)\right)\right), \\
F_{\theta}(\xi)=1-\frac{1}{2}\left(\left(1+\omega_{+}\right) \exp \left(-\gamma_{-}^{\left(\mu_{+}\right)} \xi\right)+\left(1-\omega_{+}\right) \exp \left(-\gamma_{+}^{\left(\mu_{+}\right)} \xi\right)\right), \\
\mathscr{F}(\xi)=\int_{0}^{\xi} F_{r}\left(\xi^{\prime}\right) \mathrm{d} \xi^{\prime} .
\end{gathered}
$$

Note that, in (A 5), Re denotes the real part.

\section{Appendix B. Completion of the geostrophic amplitude equations}

As noted in the main text, asymptotic evaluation of the integrals on the right-handside of (3.11) involves lengthy analysis and the objective of this Appendix is to outline the procedure and give the results. Details can be found in Appendices F to $\mathrm{H}$.

For future reference, the solution of (3.3) is

$$
\begin{aligned}
B_{\mu}^{[3]}= & \mathscr{B}_{\mu} \exp \left(-\mathrm{i} \omega^{(\mu)} t\right)-\sum_{\nu=\mu_{+}, \mu_{+}^{*}} C_{\mu \nu} A_{\nu}\left\{\frac{\omega^{(\nu)}-\omega_{0}}{\omega^{(\nu)}-\omega^{(\mu)}-\omega_{0}} \exp \left(-\mathrm{i}\left(\omega^{(\nu)}-\omega_{0}\right) t\right)\right. \\
& \left.+\frac{\omega^{(\nu)}+\omega_{0}}{\omega^{(\nu)}-\omega^{(\mu)}+\omega_{0}} \exp \left(-\mathrm{i}\left(\omega^{(\nu)}+\omega_{0}\right) t\right)\right\}-\mathrm{i} \varepsilon^{-1} \overline{R e}-1 / 2 \sum_{\nu=\mu_{+}, \mu_{+}^{*}} \frac{D_{\mu \nu}}{\omega^{(\nu)}-\omega^{(\mu)}} A_{\nu} \\
& \times \exp \left(-\mathrm{i} \omega^{(\nu)} t\right)-2 \sum_{\substack{\lambda \\
\nu=\mu_{+}, \mu_{+}^{*}}} \frac{\Lambda_{\mu \nu \lambda}}{\omega^{(\nu)}+\omega^{(\lambda)}-\omega^{(\mu)}} A_{\nu} \mathscr{A}_{\lambda} \exp \left(-\mathrm{i}\left(\omega^{(\nu)}+\omega^{(\lambda)}\right) t\right) \\
& -\frac{1}{2} \sum_{\substack{\nu_{1}, \nu_{2}, \nu_{3}=\\
\mu_{+}, \mu_{+}^{*}}} \frac{F_{\mu \nu_{1} \nu_{2} \nu_{3}}}{\omega^{\left(\nu_{1}\right)}+\omega^{\left(\nu_{2}\right)}+\omega^{\left(\nu_{3}\right)}-\omega^{(\mu)}} A_{\nu_{1}} A_{\nu_{2}} A_{\nu_{3}} \exp \left(-\mathrm{i}\left(\omega^{\left(\nu_{1}\right)}+\omega^{\left(\nu_{2}\right)}+\omega^{\left(\nu_{3}\right)}\right) t\right),
\end{aligned}
$$

where $\mathscr{B}_{\mu}(T)$ is a slowly varying amplitude and the secular terms identified while deriving (3.7) and its conjugate should be excluded from the sums when $\mu=\mu_{+}$and $\mu=\mu_{+}^{*}$ to avoid divisions by zero.

\section{B.1. Determination of the integrals in (3.11)}

Evaluation of the surface integral in (3.11) involves boundary-layer analysis of the mean flow $\langle\boldsymbol{u}\rangle$ (see Appendix F). The result is

$$
\varepsilon^{-2} \overline{R e}^{-1} \int_{Z=0, h_{0}} \boldsymbol{u}^{(\sigma) *} \cdot(\boldsymbol{n} \cdot \nabla\langle\boldsymbol{u}\rangle) \mathrm{d}^{2} \boldsymbol{X} \sim-\varepsilon^{-1} \overline{R e}^{-1 / 2} D_{\sigma \sigma} \mathscr{A}_{\sigma}+\varepsilon^{-1} \overline{R e}^{-1 / 2} \Gamma_{\sigma}^{(1)}\left|A_{+}\right|^{2},
$$

where the first term on the right-hand side expresses modal damping by endwall viscous friction (the sidewalls do not contribute to the damping of geostrophic modes) and the final term is the first of several nonlinear contributions having the same form, $\varepsilon^{-1} \overline{R e}^{-1 / 2}\left|A_{+}\right|^{2}$, but differing numerical coefficients $\Gamma_{\sigma}$. These terms represent mean forcing of the geostrophic flow by the primary modes owing to nonlinearity, forcing which requires viscosity for its existence, as is apparent from the factor $\overline{R e}^{-1 / 2}$. 
The volume integral in (3.11) is more difficult to evaluate. Equations (2.1) and (2.6) give

$$
\int \boldsymbol{u} \cdot(\boldsymbol{u} \cdot \nabla) \boldsymbol{u}^{(\sigma) *} \mathrm{~d}^{3} \boldsymbol{X}=\mathrm{i} \sum_{\lambda_{1}, \lambda_{2}} \Lambda_{\sigma \lambda_{1} \lambda_{2}} B_{\lambda_{1}} B_{\lambda_{2}}
$$

and it is tempting to employ (2.3) directly to extend the expansion (2.5) to $O\left(\varepsilon^{2}\right)$. However, this approach runs into difficulties for the following reasons. Note first that the boundary layers in $\boldsymbol{u}$ give only $O\left(\varepsilon^{3 / 2}\right)$ contributions to the integral in (I.3.8) and hence are not present in the first two terms of (2.3), first making themselves felt in $B_{\mu}^{[3]}$. Now, the construction of layers of thickness $O(\varepsilon)$ from a modal sum such as (2.1) requires modes of wavelengths comparable with $\varepsilon$, i.e. high-order modes with $K^{(\mu)}=O\left(\varepsilon^{-1}\right)$ (specifically, large axial wavenumber $m_{\mu} \pi / h_{0}=O\left(\varepsilon^{-1}\right)$ for the endwall layers and large transverse wavenumber $k^{(\mu)}=O\left(\varepsilon^{-1}\right)$ for the sidewall layer). As $\varepsilon \rightarrow 0$, more and more such high-order $B_{\mu}^{[3]}$ are required to represent the boundary layers and, if (2.3) is used in (B 3), an asymptotically large number of terms such as $\mathrm{i} \varepsilon^{3} \Lambda_{\sigma \lambda_{1} \lambda_{2}} B_{\lambda_{1}}^{[3]} B_{\lambda_{2}}^{[3]}$, each of $O\left(\varepsilon^{3}\right)$, sum to yield a net contribution of $O\left(\varepsilon^{2}\right)$, the order to which we are working. What is more, equation (B 1 ) (giving $B_{\mu}^{[3]}$ ) is not uniformly valid up to such high orders because, when $K^{(\mu)}=O\left(\varepsilon^{-1}\right)$, the volumetric viscous term is promoted to leading order in the asymptotic analysis. Evidently a more careful approach is required.

Define $\tilde{\boldsymbol{u}}$ and $\hat{\boldsymbol{u}}$ by

$$
\tilde{\boldsymbol{u}}=\sum_{\mu}\left(\varepsilon^{1 / 2} B_{\mu}^{[1]}+\varepsilon B_{\mu}^{[2]}\right) \boldsymbol{u}^{(\mu)}, \quad \hat{\boldsymbol{u}}=\boldsymbol{u}-\tilde{\boldsymbol{u}}=\sum_{\mu} \hat{B}_{\mu} \boldsymbol{u}^{(\mu)},
$$

so that $\boldsymbol{u}=\tilde{\boldsymbol{u}}+\hat{\boldsymbol{u}} . \tilde{\boldsymbol{u}}$ consists of the first two orders of the expansion (2.3) and so has no boundary layers, giving the flow outside the layers correct to $O(\varepsilon)$, whereas $\hat{\boldsymbol{u}}$ represents the correction to $\tilde{\boldsymbol{u}}$ from all higher orders and contains the boundary layers. Writing

$$
\begin{aligned}
\int \boldsymbol{u} \cdot(\boldsymbol{u} \cdot \nabla) \boldsymbol{u}^{(\sigma) *} \mathrm{~d}^{3} \boldsymbol{X}= & \int \tilde{\boldsymbol{u}} \cdot(\tilde{\boldsymbol{u}} \cdot \nabla) \boldsymbol{u}^{(\sigma) *} \mathrm{~d}^{3} \boldsymbol{X}+\int(\tilde{\boldsymbol{u}} \cdot(\hat{\boldsymbol{u}} \cdot \nabla)+\hat{\boldsymbol{u}} \cdot(\tilde{\boldsymbol{u}} \cdot \nabla)) \boldsymbol{u}^{(\sigma) *} \mathrm{~d}^{3} \boldsymbol{X} \\
& +\int \hat{\boldsymbol{u}} \cdot(\hat{\boldsymbol{u}} \cdot \nabla) \boldsymbol{u}^{(\sigma) *} \mathrm{~d}^{3} \boldsymbol{X}
\end{aligned}
$$

each of the integrals on the right-hand side is treated separately. In (B 4), note that $\hat{B}_{\mu}=O\left(\varepsilon^{3}\right)$ because the first two orders have been subtracted in the definition $\hat{\boldsymbol{u}}=\boldsymbol{u}-\tilde{\boldsymbol{u}}$. Furthermore, our earlier analysis shows that, provided $\mu$ is not of such high-order that the non-uniformity discussed above occurs, $\hat{B}_{\mu}$ has the expansion $\hat{B}_{\mu} \sim \varepsilon^{3} B_{\mu}^{[3]}$, with $B_{\mu}^{[3]}$ given by (B 1$)$.

Using (2.6) and the definition (B 4) of $\tilde{\boldsymbol{u}}$, we have

$$
\int\left\langle\tilde{\boldsymbol{u}} \cdot(\tilde{\boldsymbol{u}} \cdot \nabla) \boldsymbol{u}^{(\sigma)^{*}}\right\rangle \mathrm{d}^{3} \boldsymbol{X}=\mathrm{i} \sum_{\lambda_{1}, \lambda_{2}} \Lambda_{\sigma \lambda_{1} \lambda_{2}}\left(\varepsilon\left\langle B_{\lambda_{1}}^{[1]} B_{\lambda_{2}}^{[1]}\right\rangle+2 \varepsilon^{3 / 2}\left\langle B_{\lambda_{1}}^{[1]} B_{\lambda_{2}}^{[2]}\right\rangle+\varepsilon^{2}\left\langle B_{\lambda_{1}}^{[2]} B_{\lambda_{2}}^{[2]}\right\rangle\right)
$$

which is evaluated (see Appendix G) using (2.4) and (3.2) and looking for terms which make a non-zero contribution to the sum. The result is

$$
\varepsilon^{-2} \int\left\langle\tilde{\boldsymbol{u}} \cdot(\tilde{\boldsymbol{u}} \cdot \nabla) \boldsymbol{u}^{(\sigma) *}\right\rangle \mathrm{d}^{3} \boldsymbol{X}=\mathrm{i} \sum_{\lambda_{1}, \lambda_{2} \in M_{g}} \Lambda_{\sigma \lambda_{1} \lambda_{2}} \mathscr{A}_{\lambda_{1}} \mathscr{A}_{\lambda_{2}}
$$


where $M_{g}$ denotes the set of all geostrophic modes and the right-hand side comes from the term $\mathscr{A}_{\mu} e^{-i \omega^{(\mu)} t}$ in (3.2) via $\left\langle B_{\lambda_{1}}^{[2]} B_{\lambda_{2}}^{[2]}\right\rangle$ in (B 6).

Using (2.6), (B 4) for $\hat{\boldsymbol{u}}$, and the leading-order expression $\tilde{\boldsymbol{u}} \sim$ $\varepsilon^{1 / 2} \sum_{\nu=\mu_{+}, \mu_{+}^{*}} A_{\nu} \exp \left(-\mathrm{i} \omega^{(v)} t\right) \boldsymbol{u}^{(v)}$ for $\tilde{\boldsymbol{u}}$, the second integral on the right-hand side of (B 5) is expressed as

$$
\int\left\langle(\tilde{\boldsymbol{u}} \cdot(\hat{\boldsymbol{u}} \cdot \nabla)+\hat{\boldsymbol{u}} \cdot(\tilde{\boldsymbol{u}} \cdot \nabla)) \boldsymbol{u}^{(\sigma)^{*}}\right\rangle \mathrm{d}^{3} \boldsymbol{X} \sim 2 \mathrm{i} \varepsilon^{1 / 2} \sum_{\substack{\lambda \\ \nu=\mu_{+}, \mu_{+}^{*}}} \Lambda_{\sigma \nu \lambda} A_{\nu}\left\langle\hat{B}_{\lambda} \exp \left(-\mathrm{i} \omega^{(\nu)} t\right)\right\rangle .
$$

It turns out (see Appendix $G$ ) that the high-order modes in $\hat{\boldsymbol{u}}$, which represent the boundary layers, make negligible contributions to the sum in (B 8). As a result, the high-order non-uniformity of the asymptotics noted above is avoided and (B 8) gives

$$
\int\left\langle(\tilde{\boldsymbol{u}} \cdot(\hat{\boldsymbol{u}} \cdot \nabla)+\hat{\boldsymbol{u}} \cdot(\tilde{\boldsymbol{u}} \cdot \nabla)) \boldsymbol{u}^{(\sigma) *}\right\rangle \mathrm{d}^{3} \boldsymbol{X} \sim 2 \mathrm{i} \varepsilon^{2} \sum_{\substack{\lambda \\ \nu=\mu_{+}, \mu_{+}}} \Lambda_{\sigma \nu \lambda} A_{\nu}\left\langle B_{\lambda}^{[3]} \exp \left(-\mathrm{i} \omega^{(\nu)} t\right)\right\rangle,
$$

with $B_{\lambda}^{[3]}$ given by (B 1) and where $\hat{B}_{\lambda} \sim \varepsilon^{3 / 2} B_{\lambda}^{[3]}$ has been used. Analysis of this result (see Appendix $\mathrm{G}$ ), looking for non-zero terms, leads to

$$
\begin{gathered}
\varepsilon^{-2} \int\left\langle(\tilde{\boldsymbol{u}} \cdot(\hat{\boldsymbol{u}} \cdot \nabla)+\hat{\boldsymbol{u}} \cdot(\tilde{\boldsymbol{u}} \cdot \nabla)) \boldsymbol{u}^{(\sigma) *}\right\rangle \mathrm{d}^{3} \boldsymbol{X} \sim \mathrm{i}\left|A_{+}\right|^{2} \sum_{\lambda \in M_{g}} \Xi_{\sigma \lambda} \mathscr{A}_{\lambda} \\
+\mathrm{i} C\left(\Lambda_{\sigma \mu_{+} \mu_{+}} A_{+}^{2} \exp (\mathrm{i} \Delta T)+\Lambda_{\sigma \mu_{+}^{*} \mu_{+}^{*}} A_{+}^{* 2} \exp (-\mathrm{i} \Delta T)\right)+\varepsilon^{-1} \overline{R e}^{-1 / 2} \Gamma_{\sigma}^{(2)}\left|A_{+}\right|^{2},
\end{gathered}
$$

where the last two terms stem from the piston-motion and viscous contributions in (B1), while the first term on the right comes from the sum in (B1) involving $\mathscr{A}_{\lambda}$. The second term represents mean nonlinear forcing of the geostrophic flow by the primary modes and requires piston motion (rather than viscosity, which is required by the final term) as a catalyst.

The sum of the right-hand sides of (B 6) and (B9) is precisely what would be obtained by formal extension of (2.5) using (2.3) in (B3) (the direct approach discounted earlier), but there remains the third integral on the right-hand side of (B 5). It is here that more care is needed because the use of a modal sum would require many high-order terms for which (B 1) does not apply. Fortunately, it is found that the integral is dominated by the endwall boundary layers and using the leading-order expression for $\boldsymbol{u}$ in those layers (see Appendix $\mathrm{H}$ ) gives

$$
\varepsilon^{-2} \int\left\langle\hat{\boldsymbol{u}} \cdot(\hat{\boldsymbol{u}} \cdot \nabla) \boldsymbol{u}^{(\sigma) *}\right\rangle \mathrm{d}^{3} \boldsymbol{X} \sim \varepsilon^{-1} \overline{\operatorname{Re}}^{-1 / 2} \Gamma_{\sigma}^{(3)}\left|A_{+}\right|^{2}
$$

as the final contribution to the geostrophic amplitude equation

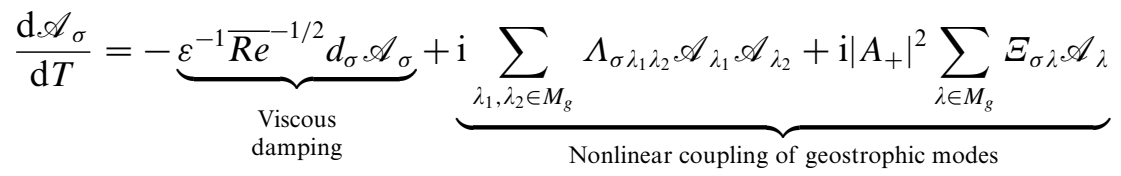

$$
\begin{aligned}
& +\underbrace{\mathrm{i} C\left(\Lambda_{\sigma \mu_{+} \mu_{+}} A_{+}^{2} \exp (\mathrm{i} \Delta T)+\Lambda_{\sigma \mu_{+}^{*} \mu_{+}^{*}} A_{+}^{* 2} \exp (-\mathrm{i} \Delta T)\right)+\varepsilon^{-1} \overline{R e}^{-1 / 2} \Gamma_{\sigma}\left|A_{+}\right|^{2}}_{\text {Nonlinear forcing of geostrophic modes }},
\end{aligned}
$$


where the right-hand side is the sum of (B 2), (B 7), (B 10), (B 11) and the volumetric viscous term from (3.11). Here, $d_{\sigma}$, given by (I.4.10), is the damping factor of mode $\sigma$ and $\Gamma_{\sigma}=\Gamma_{\sigma}^{(1)}+\Gamma_{\sigma}^{(2)}+\Gamma_{\sigma}^{(3)}$ is the sum of the three coefficients multiplying similar terms in (B 2), (B 10) and (B 11). $\Gamma_{\sigma}$ and $\Xi_{\sigma \lambda}$ are real coefficients, dependent only on $h_{0}$ and the choice of primary mode. They have the properties that $\Gamma_{\sigma}=0$ unless $n_{\sigma}=0$ and $\Xi_{\sigma \lambda}=0$ if either $n_{\sigma}=0$ or $n_{\lambda}=0$.

The damping term in (B 12) expresses a combination of endwall friction and (volumetric) viscous effects outside the boundary layers, both of which tend to slow the geostrophic flow. The second term represents nonlinearity of the geostrophic flow itself and is exactly what we would expect from the convective term in the Navier-Stokes equations if the geostrophic flow were the whole flow. Despite being nonlinear overall, the third term is linear in the $\mathscr{A}_{\mu}$ and corresponds to a coupling of geostrophic modes induced by the presence of the primary mode. The final two terms in (B 12) are both zero unless $n_{\sigma}=0$ (thanks to (2.7) and $\Gamma_{\sigma}=0$ for $n_{\sigma} \neq$ 0 ) and represent mean axisymmetric forcing of the leading-order geostrophic flow $\boldsymbol{u}^{g}=\varepsilon \sum_{\mu \in M_{g}} \mathscr{A}_{\mu} \boldsymbol{u}^{(\mu)}$ by the primary mode, forcing which arises from nonlinearity, catalysed by piston motion and viscosity. Note that, in defining the geostrophic flow as $\boldsymbol{u}^{g}=\varepsilon \sum_{\mu \in M_{g}} \mathscr{A}_{\mu} \boldsymbol{u}^{(\mu)}$, the forced contribution in (3.2) (which oscillates at frequency $\left.2 \omega_{+}\right)$for geostrophic $\mu$ has not been included. Thus, $\boldsymbol{u}^{g}$ is really the mean part of the geostrophic flow, though this distinction is not made in the main text. Equations (3.7) and (B 12) form a complete set of weakly nonlinear amplitude equations governing the coupled time evolution of the primary mode and geostrophic flow.

\section{B.2. Specialization to axisymmetric geostrophic flow}

Because forcing by the primary mode is axisymmetric, if the geostrophic flow is initially axisymmetric, i.e. $\mathscr{A}_{\mu}=0$ for all $\mu \in M_{g}$ with $n_{\mu} \neq 0$, it remains so at later times. For a primary mode growing from the linear regime, the $\mathscr{A}_{\mu}$ start off zero and it is thus reasonable to suppose axisymmetry of the geostrophic flow. This being said, a secondary symmetry-breaking instability might occur, in which case the geostrophic flow would spontaneously become non-axisymmetric and the full set of amplitude equations, (B 12) for all $\sigma \in M_{g}$, would be required. However, for simplicity, we suppose this is not the case and take the geostrophic flow as axisymmetric in the main text. Attention can then be restricted to $\sigma \in M$ and (B 12) becomes (3.12), where we have used $\Lambda_{\sigma \lambda_{1} \lambda_{2}}=\Xi_{\sigma \lambda}=0$ and $\Lambda_{\sigma \mu_{+}^{*} \mu_{+}^{*}}=-\Lambda_{\sigma \mu_{+} \mu_{+}}$(which follow when $\sigma, \lambda, \lambda_{1}, \lambda_{2} \in M$, as here, from (D 4) and $\Xi_{\sigma \lambda}=0$ for $n_{\sigma}=0$ or $n_{\lambda}=0$ ). Note that coupling of geostrophic modes has disappeared in going from (B 12) to (3.12).

\section{Appendix C. Non-axisymmetric primary modes}

In this Appendix, we briefly derive and discuss the amplitude equations for a pair of non-axisymmetric modes, $\mu_{+}, \mu_{-}$, of the same modal family. At $O(\varepsilon)$, we again obtain (3.1) and (3.2), but the sums over $v_{1}$ and $v_{2}$ now run over the four values $\mu_{+}, \mu_{-}, \mu_{+}^{*}$ and $\mu_{-}^{*}$ because the leading-order solution includes the mode pair and its conjugate in order to represent a real velocity field. At the next order, (3.3) holds as before: $\Psi_{\mu}^{L}$ is the sum of the right-hand sides of (I.4.5)(I.4.7), divided by $\delta \varepsilon$, and $\Psi_{\mu}^{N L}$ is given by (3.4), with the $\nu$-sums taken over $\mu_{+}, \mu_{-}, \mu_{+}^{*}$ and $\mu_{-}^{*}$. Secular terms in the equations for $\mu=\mu_{+}, \mu_{-}, \mu_{+}^{*}$ and 
$\mu_{-}^{*}$ may be picked out as before. When $\mu=\mu_{+}$, we obtain the non-secularity condition

$$
\begin{aligned}
& \frac{\mathrm{d} A_{+}}{\mathrm{d} T}=\mathrm{i} \omega_{+} C \exp (-\mathrm{i} \Delta T) A_{-}+\left(2 \mathrm{i} \sum_{\sigma \in M} \Lambda_{\mu_{+} \mu_{+} \sigma} \mathscr{A}_{\sigma}-\varepsilon^{-1} \overline{R e}-1 / 2 d_{+}\right) A_{+} \\
& +\mathrm{i}\left(G_{++}\left|A_{+}\right|^{2}+G_{+-}\left|A_{-}\right|^{2}\right) A_{+},
\end{aligned}
$$

to be compared with (3.7) for the axisymmetric case, where $C=C_{\mu_{+} \mu_{-}}=C_{\mu_{-} \mu_{+}}$and

$$
G_{++}=\frac{1}{2} F_{\mu_{+} \mu_{+} \mu_{+} \mu_{+}^{*}}+F_{\mu_{+} \mu_{+} \mu_{+}^{*} \mu_{+}}, \quad G_{+-}=F_{\mu_{+} \mu_{+} \mu_{-} \mu_{-}^{*}}+F_{\mu_{+} \mu_{+} \mu_{-}^{*} \mu_{-}}+F_{\mu_{+} \mu_{-} \mu_{-}^{*} \mu_{+}}
$$

are real-valued nonlinear coefficients. Similar reasoning for $\mu=\mu_{-}$leads to

$$
\begin{aligned}
& \frac{\mathrm{d} A_{-}}{\mathrm{d} T}=\mathrm{i} \omega_{-} C \exp (i \Delta T) A_{+}+\left(2 \mathrm{i} \sum_{\sigma \in M} \Lambda_{\mu_{-} \mu_{-} \sigma} \mathscr{A}_{\sigma}-\varepsilon^{-1} \overline{R e}^{-1 / 2} d_{-}\right) A_{-} \\
& +\mathrm{i}\left(G_{-+}\left|A_{+}\right|^{2}+G_{--}\left|A_{-}\right|^{2}\right) A_{-},
\end{aligned}
$$

where

$$
G_{--}=\frac{1}{2} F_{\mu_{-} \mu_{-} \mu_{-} \mu_{-}^{*}}+F_{\mu_{-} \mu_{-} \mu_{-}^{*} \mu_{-}}, \quad G_{-+}=F_{\mu_{-} \mu_{-} \mu_{+} \mu_{+}^{*}}+F_{\mu_{-} \mu_{-} \mu_{+}^{*} \mu_{+}}+F_{\mu_{-} \mu_{+} \mu_{+}^{*} \mu_{-}},
$$

are also real. Comparison of the nonlinear mode-pair equations (C 1) and (C 3) with their linear counterparts, (I.4.12) and (I.4.13), shows that they agree when the nonlinear terms are dropped (and $\hat{d}_{ \pm}=\varepsilon^{-1} \overline{R e}^{-1 / 2} d_{ \pm}$used), as one would expect.

As for the axisymmetric case, we need equations for the geostrophic, axisymmetric mode amplitudes $\mathscr{A}_{\sigma}$. Equation (3.11) holds as before and its right-hand side is treated using the same methods, but allowing for the existence of the four primary modes $\mu_{+}$, $\mu_{-}, \mu_{+}^{*}$ and $\mu_{-}^{*}$. Mean nonlinear forcing by the primary modes is axisymmetric despite the non-axisymmetric nature of these modes and, assuming the absence of a secondary, symmetry-breaking instability, the geostrophic flow is taken as axisymmetric. The resulting amplitude equations are

$$
\begin{array}{r}
\frac{\mathrm{d} \mathscr{A}_{\sigma}}{\mathrm{d} T}=-\varepsilon^{-1} \overline{R e}^{-1 / 2} d_{\sigma} \mathscr{A}_{\sigma}+\mathrm{i} \tilde{C}_{\sigma}\left(A_{+} A_{-}^{*} \exp (\mathrm{i} \Delta T)-A_{+}^{*} A_{-} \exp (-\mathrm{i} \Delta T)\right) \\
+\varepsilon^{-1} \overline{R e}^{-1 / 2}\left(\Gamma_{\sigma}^{+}\left|A_{+}\right|^{2}+\Gamma_{\sigma}^{-}\left|A_{-}\right|^{2}\right),
\end{array}
$$

for $\sigma \in M$, where the coefficients $\Gamma_{\sigma}^{ \pm}$and

$$
\tilde{C}_{\sigma}=2 \omega_{-} \sum_{\lambda \neq \mu_{-}^{*}} \frac{C_{\lambda \mu_{+}^{*}} \Lambda_{\sigma \mu_{-} \lambda}}{\omega_{-}+\omega^{(\lambda)}}-2 \omega_{+} \sum_{\lambda \neq \mu_{+}^{*}} \frac{C_{\lambda \mu_{-}^{*}} \Lambda_{\sigma \mu_{+} \lambda}}{\omega_{+}+\omega^{(\lambda)}},
$$

are real.

Adopting the new amplitude variables

$$
a_{+}=A_{+} \exp (\mathrm{i} \Delta T / 2), \quad a_{-}=A_{-} \exp (-\mathrm{i} \Delta T / 2)
$$

for the primary modes yields

$$
\begin{array}{r}
\frac{\mathrm{d} a_{+}}{\mathrm{d} T}=\mathrm{i} \omega_{+} C a_{-}+\left(\frac{1}{2} \mathrm{i} \Delta+2 \mathrm{i} \sum_{\sigma \in M} \Lambda_{\mu_{+} \mu_{+} \sigma} \mathscr{A}_{\sigma}-\varepsilon^{-1} \overline{R e}^{-1 / 2} d_{+}\right) a_{+} \\
+\mathrm{i}\left(G_{++}\left|a_{+}\right|^{2}+G_{+-}\left|a_{-}\right|^{2}\right) a_{+},
\end{array}
$$




$$
\begin{gathered}
\frac{\mathrm{d} a_{-}}{\mathrm{d} T}=\mathrm{i} \omega_{-} C a_{+}-\left(\frac{1}{2} \mathrm{i} \Delta-2 \mathrm{i} \sum_{\sigma \in M} \Lambda_{\mu_{-} \mu_{-} \sigma} \mathscr{A}_{\sigma}+\varepsilon^{-1} \overline{R e}{ }^{-1 / 2} d_{-}\right) a_{-}+\mathrm{i}\left(G_{-+}\left|a_{+}\right|^{2}+G_{--}\left|a_{-}\right|^{2}\right) a_{-}, \\
\frac{\mathrm{d} \mathscr{A}_{\sigma}}{\mathrm{d} T}=-\varepsilon^{-1} \overline{R e}^{-1 / 2} d_{\sigma} \mathscr{A}_{\sigma}+\mathrm{i} \tilde{C}_{\sigma}\left(a_{+} a_{-}^{*}-a_{+}^{*} a_{-}\right)+\varepsilon^{-1} \overline{R e}^{-1 / 2}\left(\Gamma_{\sigma}^{+}\left|a_{+}\right|^{2}+\Gamma_{\sigma}^{-}\left|a_{-}\right|^{2}\right) .
\end{gathered}
$$

Introducing magnitudes and phases via $a_{+}=\left|a_{+}\right| \exp -\mathrm{i}\left(\psi_{+}+\pi / 4\right)$ and $a_{-}=$ $\left|a_{-}\right| \exp -\mathrm{i}\left(\psi_{-}-\pi / 4\right)$, we obtain

$$
\begin{gathered}
\frac{\mathrm{d}\left|a_{+}\right|}{\mathrm{d} T}=-\varepsilon^{-1} \overline{R e}^{-1 / 2} d_{+}^{r}\left|a_{+}\right|-\omega_{+} C\left|a_{-}\right| \cos \psi, \\
\frac{\mathrm{d}\left|a_{-}\right|}{\mathrm{d} T}=-\varepsilon^{-1} \overline{R e}^{-1 / 2} d_{-}^{r}\left|a_{-}\right|+\omega_{-} C\left|a_{+}\right| \cos \psi, \\
\frac{\mathrm{d} \psi}{\mathrm{d} T}=C\left(\omega_{+} \frac{\left|a_{-}\right|}{\left|a_{+}\right|}-\omega_{-} \frac{\left|a_{+}\right|}{\left|a_{-}\right|}\right) \sin \psi-\hat{\Delta}+2 \sum_{\sigma \in M}\left(\Lambda_{\mu_{-} \mu_{-} \sigma}-\Lambda_{\mu_{+} \mu_{+} \sigma}\right) \mathscr{A}_{\sigma} \\
+\left(G_{-+}-G_{++}\right)\left|a_{+}\right|^{2}+\left(G_{--}-G_{+-}\right)\left|a_{-}\right|^{2},
\end{gathered}
$$

and

$$
\frac{\mathrm{d} \mathscr{A}_{\sigma}}{\mathrm{d} T}=-\varepsilon^{-1} \overline{R e}^{-1 / 2} d_{\sigma} \mathscr{A}_{\sigma}+2 \tilde{C}_{\sigma}\left|a_{+}\right|\left|a_{-}\right| \cos \psi+\varepsilon^{-1} \overline{R e}^{-1 / 2}\left(\Gamma_{\sigma}^{+}\left|a_{+}\right|^{2}+\Gamma_{\sigma}^{-}\left|a_{-}\right|^{2}\right),
$$

where $\psi=\psi_{+}-\psi_{-}$represents the difference in phases of the two primary modes. Terms on the right-hand sides of $(\mathrm{C} 8)-(\mathrm{C} 14)$ may be interpreted as for the axisymmetric case.

A number of analytical results, similar to those obtained earlier for axisymmetric primary modes, can be derived. As for the axisymmetric case, in the absence of viscosity, the system (C 11)-(C 14) is integrable and the solution is either periodic or homoclinic to a fixed point. Starting from the linear regime of infinitesimal amplitudes, the perturbation grows when the basic flow is unstable, reaches a maximum and decays back to zero, behaviour which again illustrates the singular nature of the inviscid problem. Non-zero fixed points of (C11)-(C 14) may be determined as for the axisymmetric case, with similar results. The phase difference is given by $\cos \psi=$ $-\operatorname{sgn}(C) \varepsilon_{c} / \varepsilon$, so such fixed points can only exist if $\varepsilon \geqslant \varepsilon_{c}$, where, as in Part 1, $\varepsilon_{c}=\left(-d_{+}^{r} d_{-}^{r} / \omega_{+} \omega_{-} \overline{\operatorname{Re}} C^{2}\right)^{1 / 2}$ is the critical piston amplitude from linear theory. The amplitudes $\left|a_{+}\right|$and $\left|a_{-}\right|$both vary with piston frequency in the manner of figure 4, forming parabolas which branch out from the ends of the linear instability interval $|\hat{\Delta}|<\Delta_{0}$. As before, the lower branch is always unstable, whereas the upper branch is stable near its bifurcation from the basic flow, but may lose stability elsewhere. Note that these branches do not represent fixed points in the sense that neither $a_{+}$or $a_{-}$need individually be constant. Instead, $\psi_{+}$and $\psi_{-}$are linear functions of $T$ whose difference is constant. It can be shown that a steadily rotating frame of reference (neither the inertial frame, nor the one rotating with the cylinder) can be found in which the flow field resulting from the fixed point is periodic and phase-locked to the piston motion with twice the piston period. Finally, assuming, as before, that divergence is accompanied by $\psi \rightarrow \psi_{\infty}$, it may be shown that divergence is only possible above a threshold piston amplitude, $\varepsilon_{d}$, dependent on $\overline{R e}, h_{0}$ and the choice of primary mode pair and which may be infinite (in which case divergence cannot occur no matter what the values of $\varepsilon$ and $\hat{\Delta}$ ). 
Further progress would require calculation of the nonlinear coefficients in (C13) and (C 14), a task we have not so far undertaken because of the amount of analytical and numerical work it would appear to entail. Nonetheless, we did carry out a numerical study (see Racz 2006) based on (C 11)-(C 13) without the geostrophic term. By rescaling $\left|a_{+}\right|,\left|a_{-}\right|$and $T$, the number of constants appearing in the equations can be reduced to four, which were taken as the controlling parameters of the problem, rather than the physical ones $\overline{R e}, h_{0}, \hat{\Delta}$ and $\varepsilon$. Depending upon the parameters and initial conditions, either a fixed point, periodic or aperiodic oscillations, or divergence were found at large times. This may be contrasted with the axisymmetric case, for which neglecting the effects of the geostrophic flow on the primary mode always leads to a fixed point. This is not to say that the geostrophic modes are unimportant in the non-axisymmetric case, indeed they obviously ought to be included, but the dynamics are already as rich without them as they are likely to become with their inclusion.

\section{REFERENCES}

Busse, F. H. 1968 Steady fluid flow in a precessing spheroidal shell. J. Fluid Mech. 33, 739-751.

Douady, S. 1990 Experimental study of Faraday instability. J. Fluid Mech. 221, 383-409.

Duguet, Y., Scott, J. \& Le Penven, L. 2005 Instability inside a rotating gas cylinder subject to axial periodic strain. Phys. Fluids 17, 114103.

Eloy, C., Le Gal, P. \& Le Dizés, S. 2003 Elliptic and triangular instabilities in rotating cylinders. J. Fluid Mech. 476, 357-388.

Fultz, D. 1959 A note on overstability, and the elastoid-inertia oscillations of Kelvin, Solberg and Bjerknes. J. Met. 16, 199-208.

Graftieaux, L. 2003 Etude expérimentale de l'instabilité d'un cylindre de gaz tournant soumis à une compression périodique. $\mathrm{PhD}$ thesis, Ecole Centrale de Lyon.

Graftieaux, L., Le Penven, L., Scott, J. F. \& Grosjean, N. 2002 A new parametric instability in rotating cylinder flow. In Advances in Turbulence IX (ed. I. P. Castro \& P. E. Hancock). CIMNE, Barcelona, Spain.

Greenspan, H. 1969 On the non-linear interaction of inertial modes. J. Fluid Mech. 36, 257-264.

GunN, J. S. \& AldRIDGE, K. D. 1990 Inertial wave eigenfrequencies for a non-uniformly rotating fluid. Phys. Fluids A 2(11), 2055-2060.

Kerswell, R. R. 1999 Secondary instabilities in rapidly rotating flows: inertial wave breakdown. J. Fluid Mech. 382, 283-306.

Kerswell, R. R. 2002 Elliptical instability. Annu. Rev. Fluid Mech. 34, 83-114.

McEwan, A. D. 1970 Inertial oscillations in a rotating fluid cylinder. J. Fluid Mech. 40, 603-640.

Malkus, W. V. R. 1989 An experimental study of the global instabilities due to tidal (elliptical) distortion of a rotating elastic cylinder. Geophys. Astrophys. Fluid Dyn. 48, 123-134.

ManasseH, R. 1992 Breakdown regimes of inertia waves in a precessing cylinder. J. Fluid Mech. 243, 261-296.

Mason, D. M. \& Kerswell, R. R. 1999 Nonlinear evolution of the elliptical instability: an example of inertial wave breakdown. J. Fluid Mech., 396 73-108.

Miles, J. 1993 On Faraday waves. J. Fluid Mech. 248, 671-683.

MiLner, S. T. 1991 Square patterns and secondary instabilities in driven capillary waves. J. Fluid Mech. 225, 81-100.

RACZ, J.-P. 2006 Instabilité paramétrique dans un cylindre de gaz en rotation soumis à la compression périodique d'un piston. $\mathrm{PhD}$ thesis, Ecole Centrale de Lyon.

Racz, J.-P. \& ScotT, J. F. 2008 Parametric instability in a rotating cylinder of gas subject to sinusoidal axial compression. Part 1. Linear theory. J. Fluid Mech. 595, 265-290.

WALEFFE, F. 1989 The 3D instability of a strained vortex and its relation to turbulence. PhD thesis, MIT.

WALEFFE, F. 1990 On the three-dimensional instability of strained vortices. Phys. Fluids A 2, 76-80. 